\title{
AIRY POINT PROCESS AT THE LIQUID-GAS BOUNDARY
}

\author{
By VinCEnt BefFARA ${ }^{1}$, Sunil ChHitA ${ }^{1}$ AND Kurt JOHANSSON ${ }^{2}$ \\ Université Grenoble Alpes, Durham University and \\ Royal Institute of Technology KTH
}

\begin{abstract}
Domino tilings of the two-periodic Aztec diamond feature all of the three possible types of phases of random tiling models. These phases are determined by the decay of correlations between dominoes and are generally known as solid, liquid and gas. The liquid-solid boundary is easy to define microscopically and is known in many models to be described by the Airy process in the limit of a large random tiling. The liquid-gas boundary has no obvious microscopic description. Using the height function, we define a random measure in the two-periodic Aztec diamond designed to detect the long range correlations visible at the liquid-gas boundary. We prove that this random measure converges to the extended Airy point process. This indicates that, in a sense, the liquid-gas boundary should also be described by the Airy process.
\end{abstract}

\section{Introduction.}

1.1. The two-periodic Aztec diamond and random tilings. An Aztec diamond graph of size $n$ is a bipartite graph which contains white vertices given by

$$
\mathrm{W}=\{(i, j): i \bmod 2=1, j \bmod 2=0,1 \leq i \leq 2 n-1,0 \leq j \leq 2 n\}
$$

and black vertices given by

$$
\mathrm{B}=\{(i, j): i \bmod 2=0, j \bmod 2=1,0 \leq i \leq 2 n, 1 \leq j \leq 2 n-1\} .
$$

The edges of the Aztec diamond graph are given by $\mathrm{b}-\mathrm{w}= \pm e_{1}, \pm e_{2}$ for $\mathrm{b} \in \mathrm{B}$ and $\mathrm{w} \in \mathrm{W}$, where $e_{1}=(1,1)$ and $e_{2}=(-1,1)$. The coordinate of a face in the graph is defined to be the coordinate of its center. For an Aztec diamond graph of size $n=4 m$ with $m \in \mathbb{N}$, define the two-periodic Aztec diamond to be an Aztec diamond graph with edge weights $a$ for all edges incident to the faces $(i, j)$ with $(i+j) \bmod 4=2$ and edge weights $b$ for all the edges incident to the faces $(i, j)$ with $(i+j) \bmod 4=0$; see the left figure in Figure 1 . We call the faces $(i, j)$ with $(i+j) \bmod 4=2$ to be the $a$-faces. For the purpose of this paper, we set $b=1$; this incurs no loss of generality, since multiplying both $a$ and $b$ by the same constant does not change the model that we consider.

Received February 2017; revised November 2017.

${ }^{1}$ Supported by the German Research Foundation in SFB 1060-B04 "The Mathematics of Emergent Effects".

${ }^{2}$ Supported by the Knut and Alice Wallenberg Foundation Grant KAW:2010.0063.

MSC2010 subject classifications. 60G55, 82B20, 60K35.

Key words and phrases. Domino tilings, Airy kernel point process, two-periodic Aztec diamond. 


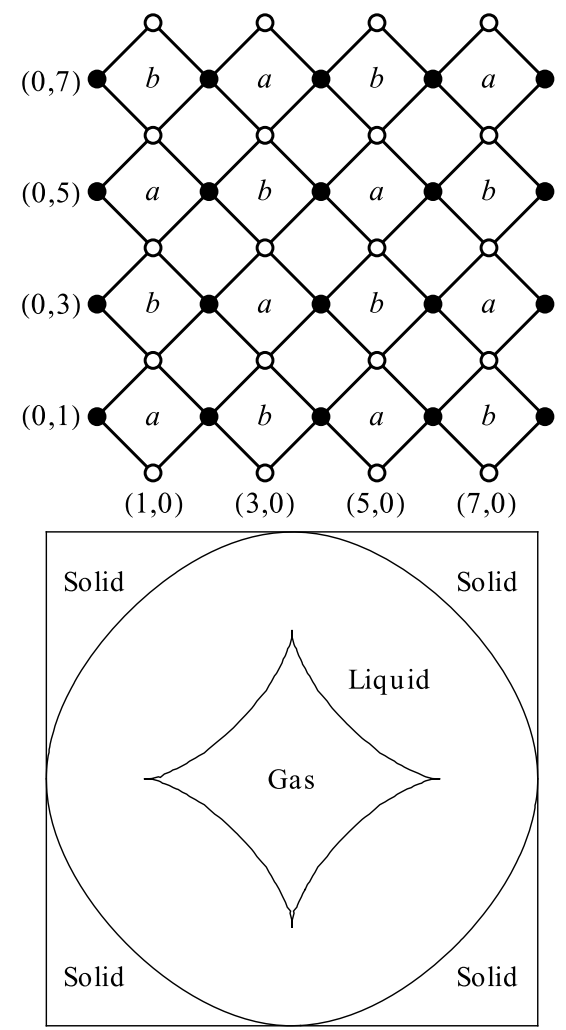

FIG. 1. The top figure shows the two-periodic Aztec diamond graph for $n=4$ with the edges weights given by $a$ (or $b)$ if the edge is incident to a face marked a (resp., $b$ ). The bottom figure shows the limit shape when $a=0.5$ and $b=1 ;$ see $[9,14,28]$ for the explicit curve.

A dimer is an edge and a dimer covering is a subset of edges so that each vertex is incident to exactly one edge. Each dimer covering of the two-periodic Aztec diamond is picked with probability proportional to the product of its edge weights. For the two-periodic Aztec diamond, it is immediate that each dimer covering is picked uniformly at random when $a=1$.

An equivalent notion to a dimer covering is a domino tiling, where one replaces each dimer by a domino. Each dimer is the graph theoretic dual of a domino. Simulations of domino tilings of large bounded regions exhibit interesting features due to the emergence of a limit shape. Here, the tiling separates into distinct macroscopic regions: solid, where the configurations of dominoes are deterministic; liquid, where the correlation between dominoes have polynomial decay in distance; and gas where the dominoes have exponential decay of correlations. These phases are characterised in [29] but first noticed in [30]. Even though their names may suggest otherwise, these regions are not associated with physical states of matter. 
An alternate convention is to say that the solid region is the frozen region while the liquid and gas regions are the unfrozen regions. The liquid region is then referred to as the rough unfrozen region (or simply rough region) and the gas region is referred to as the smooth unfrozen region.

Considerable research attention has been directed to tiling models, including domino tilings and lozenge tilings, on bounded regions whose limit shapes contain both solid and liquid regions, but no gaseous regions. The primary reason behind this attention lies in the fact that in several cases these models are mathematically tractable due to direct connections with algebraic combinatorics through the socalled Schur processes [31]. By exploiting the algebraic structure via the so-called Schur generating functions, it is possible to find the limit shape, and the local and global bulk limiting behaviours in several cases; see the recent articles [8, 20, 32]. More computational approaches are also used to find these asymptotic quantities as well as the limiting edge behaviour. These approaches often use in an essential way that the dimers, or some associated particles, form a determinantal process with an explicit correlation kernel. Finding this correlation kernel is not a simple task in general, but successful techniques have come from applying the KarlinMcGregor-Lindström-Gessel-Viennot matrix and the Eynard-Mehta theorem, or using vertex operators; see [2] for the former and $[4,5]$ for a recent exposition of the latter. The limiting correlation kernels that appear are often the same or related to those that occur in random matrix theory. Indeed, in [19, 22, 31, 33], the limiting behaviour of the random curve separating the solid and liquid regions is described by the Airy process, a universal probability distribution first appearing in [35] in connection with random growth models.

Domino tilings of the two-periodic Aztec diamond do not belong to the Schur process class. In fact, the two techniques mentioned above fail (at least for us) for this model. However, in [10], the authors derive a formula for the correlations of dominoes for the two-periodic Aztec diamond, that is, they give a formula for the so-called the inverse Kasteleyn matrix; see below for more details. The formula given in [10] is particularly long and without any specific algebraic or asymptotic structure. In [9], the formula is dramatically simplified and written in a good form for asymptotic analysis. Precise asymptotic expansions of the inverse Kasteleyn matrix reveal the limit shape as well as the asymptotic entries of the inverse Kasteleyn matrix in all three macroscopic regions, and at the solid-liquid and liquidgas boundaries. Due to technical considerations, these asymptotic computations were only performed along the main diagonal of the two-periodic Aztec diamond. Roughly speaking, the outcome is that the asymptotics of the inverse Kasteleyn matrix at the liquid-gas boundary is given by a mixture of a dominant "gas part" and a lower order "Airy part" correction. Unfortunately, these asymptotic results only describe the statistical behaviour of the dominoes at the liquid-gas boundary, and do not determine the nature of this boundary. More explicitly, it is highly plausible, as can be seen in simulations (see Figure 2) that there is a family of lattice paths which separate the liquid and gas regions. The exact microscopic definition 


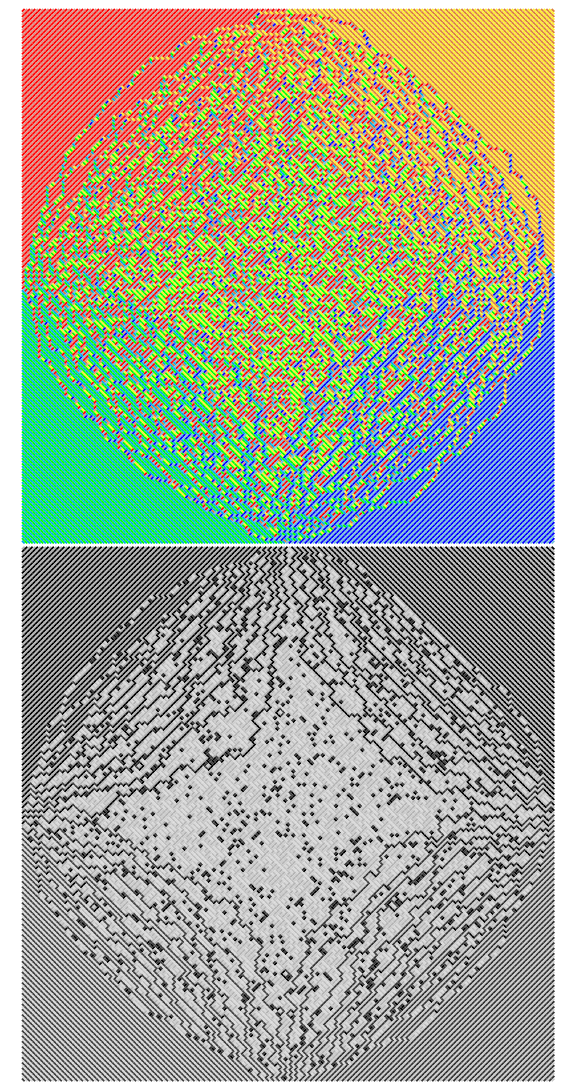

FIG. 2. Two different drawings of a simulation of a domino tiling of the two-periodic Aztec diamond of size 200 with $a=0.5$ and $b=1$. The top figure contains eight different colours, highlighting the solid and liquid phases. The bottom figure contains eight different gray-scale colours to accentuate the gas phase. We choose $a=0.5$ for aesthetic reasons in relation of the size of the Aztec diamond and the size of the liquid and gas regions.

of these paths is not clear; see [9] for a discussion and a suggestion. The asymptotic computations in [9] do not give us any information about these paths. At the liquid-solid boundary, the definition of the boundary is obvious; it is the first place where we see a deviation from the regular brick wall pattern. At the liquid-gas boundary, however, these paths, if they exist, are in some sense "sitting" in a "gas" background. The paths represent long-distance correlations and the purpose of this paper is to extract these correlations from the background "gas noise" and show that they are described by the Airy point process. This strongly indicates that there should be a random boundary path at the liquid-gas boundary which, appropriately rescaled, converges to the Airy process just as at the liquid-solid boundary.

We approach the problem via the so-called height function of the domino tiling, an idea originally introduced by Thurston [36]. The height function is defined for 
the two-periodic Aztec diamond at the center of each face of the Aztec diamond graph, characterised by the height differences in the following way: if there is a dimer covering the edge shared between two faces, the height difference between the two faces is \pm 3 , while if there is no dimer covering the shared edge between two faces the height difference is $\mp 1$. We use the convention that as we traverse from one face to an adjacent face, the height difference will be +3 if there is a dimer covering the shared edge and the left vertex of the incident edge is black. Similarly, the height difference is +1 when we cross an empty edge with a white vertex to the left. We assign the height at the face $(0,0)$ (outside of the Aztec diamond graph) to be equal to 0 . The height function on the faces bordering the Aztec diamond graph are deterministic and given by the above rule. Figure 3 shows a domino tiling of the Aztec diamond with the heights labeled at each face.

The height function has a limit shape that is the solution of a certain variational problem and this also, in principle, leads to a description of the macroscopic boundaries between the regions with different phases [11, 28]. For the two-periodic

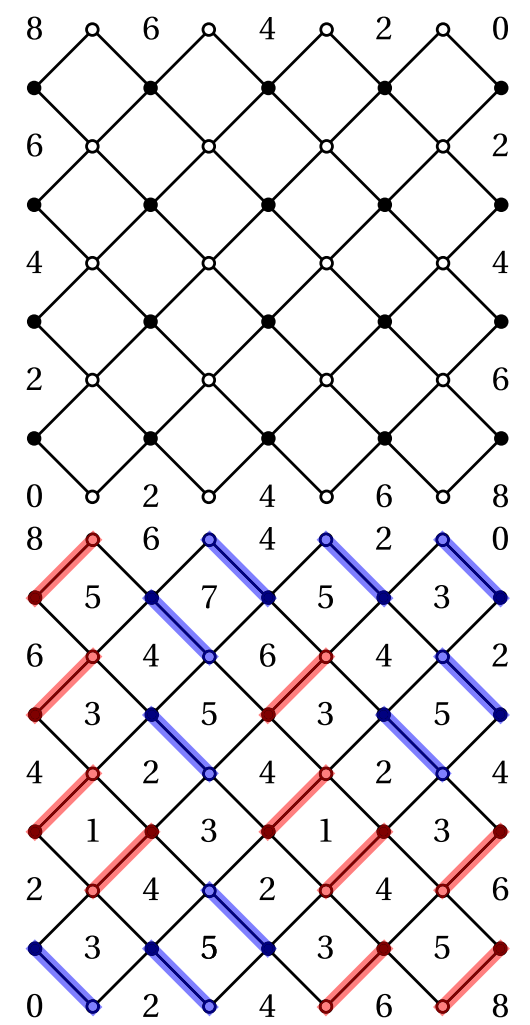

FIG. 3. The top figure shows the height function of an Aztec diamond graph of size 4 on the bordering faces imposing that the height at face $(0,0)$ is 0 . The bottom figure shows the same graph with a dimer covering and its corresponding height function. 
Aztec diamond, this gives the algebraic equation for the boundaries seen in Figure 1. The solid and gas phases correspond to flat pieces, facets, of the limit shape. The solid phase has a completely flat height function even at the microscopic level, whereas the height function in the gas phase has small fluctuations. The global fluctuations of the height function in the liquid, or rough phase, for many tilings models have been studied revealing the so-called Gaussian-free field in the limit; see the papers $[1,3,7,13,15-18,25,26,34]$ for examples with varying techniques of proof. Hence, the liquid-gas interface can be seen as an example of a boundary between a rough random crystal surface and a facet with small, random, almost independent and Poissonnian dislocations.

The novelty of this paper is that we use the height function close to the liquidgas boundary to introduce a random measure, defined in detail below, which captures the long distance changes in the height function, but averages out the local height fluctuations coming from the surrounding gas phase. This random measure gives a partial explanation of the nature of the liquid-gas boundary.

1.2. Definition of the random measure. Let $\mathbb{I}_{A}$ be the indicator function for some set $A$ and denote $\mathbb{I}$ to be the identity matrix or operator. Let $\mathrm{Ai}(\cdot)$ denote the standard Airy function, and define

$$
\tilde{\mathcal{A}}\left(\tau_{1}, \zeta_{1} ; \tau_{2}, \zeta_{2}\right)=\int_{0}^{\infty} e^{-\lambda\left(\tau_{1}-\tau_{2}\right)} \operatorname{Ai}\left(\zeta_{1}+\lambda\right) \operatorname{Ai}\left(\zeta_{2}+\lambda\right) d \lambda
$$

and

$$
\phi_{\tau_{1}, \tau_{2}}\left(\zeta_{1}, \zeta_{2}\right)=\mathbb{I}_{\tau_{1}<\tau_{2}} \frac{1}{\sqrt{4 \pi\left(\tau_{2}-\tau_{1}\right)}} e^{-\frac{\left(\zeta_{1}-\zeta_{2}\right)^{2}}{4\left(\tau_{2}-\tau_{1}\right)}-\frac{\left(\tau_{2}-\tau_{1}\right)\left(\zeta_{1}+\zeta_{2}\right)}{2}+\frac{\left(\tau_{2}-\tau_{1}\right)^{3}}{12}},
$$

the latter is referred to as the Gaussian part of the extended Airy kernel; see [21]. The extended Airy kernel, $\mathcal{A}\left(\tau_{1}, \zeta_{1} ; \tau_{2}, \zeta_{2}\right)$, is defined by

$$
\mathcal{A}\left(\tau_{1}, \zeta_{1} ; \tau_{2}, \zeta_{2}\right)=\tilde{\mathcal{A}}\left(\tau_{1}, \zeta_{1} ; \tau_{2}, \zeta_{2}\right)-\phi_{\tau_{1}, \tau_{2}}\left(\zeta_{1}, \zeta_{2}\right) \text {. }
$$

Let $\beta_{1}<\cdots<\beta_{L_{1}}, L_{1} \geq 1$, be given fixed real numbers. The extended Airy kernel gives a determinantal point process on $L_{1}$ lines $\left\{\beta_{1}, \ldots, \beta_{L_{1}}\right\} \times \mathbb{R}$. We think of this process as a random measure $\mu_{\mathrm{Ai}}$ on $\left\{\beta_{1}, \ldots, \beta_{L_{1}}\right\} \times \mathbb{R}$ in the following way.

Let $A_{1}, \ldots, A_{L_{2}}, L_{2} \geq 1$, be finite, disjoint intervals in $\mathbb{R}$ and write

$$
\Psi(x)=\sum_{p=1}^{L_{2}} \sum_{q=1}^{L_{1}} w_{p, q} \mathbb{I}_{\left\{\beta_{q}\right\} \times A_{p}}(x)
$$

for $x \in\left\{\beta_{1}, \ldots, \beta_{L_{1}}\right\} \times \mathbb{R}$, where $w_{p, q}$ are given complex numbers. Then

$$
\begin{aligned}
\mathbb{E}\left[\exp \left(\sum_{p=1}^{L_{2}} \sum_{q=1}^{L_{1}} w_{p, q} \mu_{\mathrm{Ai}}\left(\left\{\beta_{q}\right\} \times A_{p}\right)\right)\right] \\
=\operatorname{det}\left(\mathbb{I}+\left(e^{\Psi}-1\right) \mathcal{A}\right)_{L^{2}\left(\left\{\beta_{1}, \ldots, \beta_{L_{1}}\right\} \times \mathbb{R}\right)}
\end{aligned}
$$

for $w_{p, q} \in \mathbb{C}$, defines the random measure $\mu_{\mathrm{Ai}}$, the extended Airy point process. 
The positions of the particles in the extended Airy point process can be thought of as the intersections of the lines $\left\{\beta_{1}, \ldots, \beta_{L_{1}}\right\} \times \mathbb{R}$ with a family of random curves (a line ensemble; see [12]). If we think of these lines as level lines of some height function, then $\mu_{\mathrm{Ai}}\left(\left\{\beta_{q}\right\} \times A\right)$ is the height change in $A$ along the line $\left\{\beta_{q}\right\} \times A$. We want to define a random measure in a random tiling of the two-periodic Aztec diamond close to the liquid-gas boundary which captures the long distance height differences, and which converges to $\mu_{\mathrm{Ai}}$. Take $L_{1}$ lines in the Aztec diamond at distances of order $m^{2 / 3}$ from each other, and look at the height differences along these lines in intervals of length $O\left(\mathrm{~m}^{1 / 3}\right)$ close to the liquid-gas boundary. In Figure 2, we see something like long random curves, but the height differences along an interval in the diamond will come not only from these curves but also from the smaller sized objects which are in a sense due to the gas-like features in the background. We expect that these smaller sized objects are much smaller than $O\left(\mathrm{~m}^{1 / 3}\right)$, and we further expect that the correlations between these smaller sized objects decay rapidly. We isolate the effects of the long curves by taking averages of the height differences along copies of the intervals on $M$ parallel lines, with $M$ tending to infinity slowly as $m$ tends to infinity. The distances between the copies are of order $(\log m)^{2}$, which is less than $m^{2 / 3}$, but large enough for the short range correlations to decay. We will now make these ideas precise and define a random measure on $\mu_{m}$ on $\left\{\beta_{1}, \ldots, \beta_{L_{1}}\right\} \times \mathbb{R}$ that we will show converges to $\mu_{\mathrm{Ai}}$.

The following constants come from the asymptotic results for the inverse Kasteleyn matrix for the two-periodic Aztec diamond; see [9] and Theorem 6.1 below. Let

$$
c=\frac{a}{\left(1+a^{2}\right)}
$$

which occurs throughout the paper. For this paper, we fix $\xi=-\frac{1}{2} \sqrt{1-2 c}$ and set

$$
c_{0}=\frac{(1-2 c)^{\frac{2}{3}}}{(2 c(1+2 c))^{\frac{1}{3}}}, \quad \lambda_{1}=\frac{\sqrt{1-2 c}}{2 c_{0}} \quad \text { and } \quad \lambda_{2}=\frac{(1-2 c)^{\frac{3}{2}}}{2 c c_{0}^{2}} .
$$

The term $\xi$ can be thought of as the asymptotic parameter which puts the analysis at the liquid-gas boundary after rescaling (along the main diagonal in the third quadrant of the rotated Aztec diamond). The terms $\lambda_{1}$ and $\lambda_{2}$ are scale parameters, as found in [9].

We will define discrete lines $\mathcal{L}_{m}(q, k), q \in\left\{1, \ldots, L_{1}\right\}, 1 \leq k \leq M$, which we should think of as $M$ copies of the lines $\left\{\beta_{1}, \ldots, \beta_{L_{1}}\right\} \times \mathbb{R}$ embedded in the Aztec diamond as mentioned above. Recall that $e_{1}=(1,1)$ and $e_{2}=(-1,1)$. Set

$$
\beta_{m}(q, k)=2\left[\beta_{q} \lambda_{2}(2 m)^{2 / 3}+k \lambda_{2}(\log m)^{2}\right]
$$

and define

$$
\mathcal{L}_{m}(q, k)=\mathcal{L}_{m}^{0}(q, k) \cup \mathcal{L}_{m}^{1}(q, k),
$$




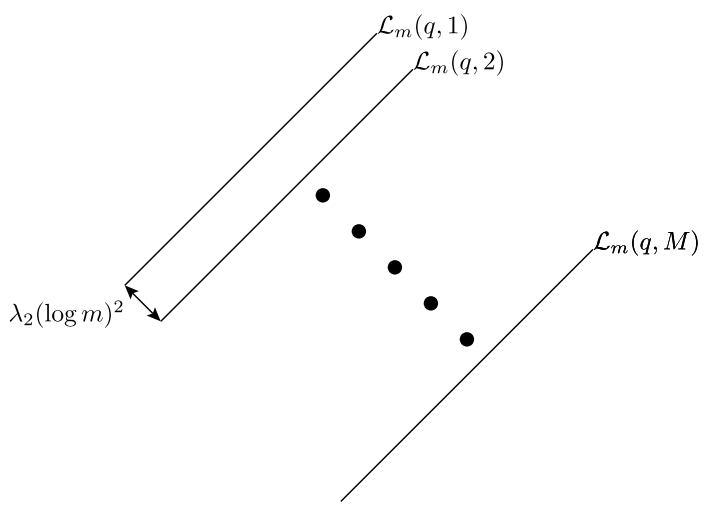

FIG. 4. The lines $\mathcal{L}_{m}(q, 1)$ to $\mathcal{L}_{m}(q, M)$.

where, for $\varepsilon \in\{0,1\}$,

$$
\mathcal{L}_{m}^{\varepsilon}(q, k)=\left\{\left(2 t-\varepsilon+\frac{1}{2}\right) e_{1}-\beta_{m}(q, k) e_{2} ; t \in[0,4 m] \cap \mathbb{Z}\right\} .
$$

The lines $\mathcal{L}_{m}(q, k)$ are discrete lines parallel to the main diagonal with direction $(1,1)$; see Figure 4. Write

$$
\mathcal{L}_{m}^{\varepsilon}=\bigcup_{q=1}^{L_{1}} \bigcup_{k=1}^{M} \mathcal{L}_{m}^{\varepsilon}(q, k)
$$

and

$$
\mathcal{L}_{m}=\mathcal{L}_{m}^{0} \cup \mathcal{L}_{m}^{1},
$$

so that $\mathcal{L}_{m}$ is the union of all these discrete lines. For $z \in \mathcal{L}_{m}$, we write

$$
\varepsilon(z)=\varepsilon \quad \text { if } z \in \mathcal{L}_{m}^{\varepsilon} .
$$

Each of the points in $\mathcal{L}_{m}^{\varepsilon}$ may be covered by a dimer. When computing height differences, the sign of the height change as we cross a dimer depends on whether $\varepsilon=0$ or 1 . Later, we will think of these dimers as particles and $\varepsilon$ will then be called the parity of the particle. We think of $\varepsilon(z)=0$ having even parity while $\varepsilon(z)=1$ having odd parity.

We call a subset $I \subseteq \mathcal{L}_{m}(q, k)$ a discrete interval if it has the form

$$
I=\left\{\left(\frac{1}{2}+t\right) e_{1}-\beta_{m}(q, k) e_{2} ; t_{1} \leq t<t_{2}\right\},
$$

where $t_{1}, t_{2} \in 2 \mathbb{Z}+1$. We denote the height of the face $F$ by $h(F)$ as defined in Section 1.1. The $a$-faces adjacent to the discrete interval $I$ in (1.6) are defined to be the faces

$$
\begin{aligned}
& F_{+}(I)=t_{2} e_{1}-\beta_{m}(q, k) e_{2}, \\
& F_{-}(I)=t_{1} e_{1}-\beta_{m}(q, k) e_{2},
\end{aligned}
$$

which are the end faces of a discrete interval; see Figure 5. 


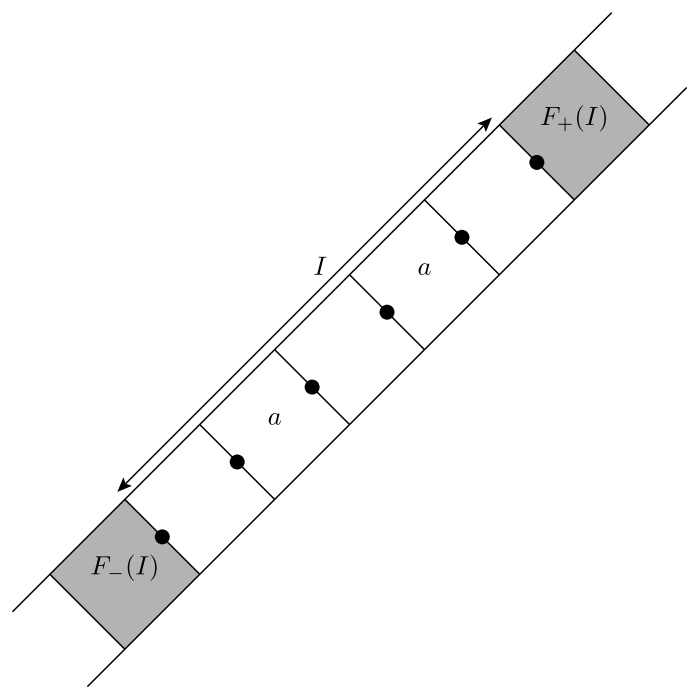

FIG. 5. The two endpoints of $I: F_{-}(I)$ and $F_{+}(I)$.

The height difference along $I$ is then

$$
\Delta h(I)=h\left(F_{+}(I)\right)-h\left(F_{-}(I)\right) .
$$

Write

$$
\rho_{m}=4[m(1+\xi)], \quad \tau_{m}(q)=\left[\beta_{q}^{2} \lambda_{1}(2 m)^{1 / 3}\right] .
$$

We want to embed the real line intervals $A_{p}=\left[\alpha_{p}^{l}, \alpha_{p}^{r}\right], 1 \leq p \leq L_{2}$, in the Aztec diamond as discrete intervals close to the liquid-gas boundary. For this, and for the asymptotic analysis later in the paper, it is convenient to use the following parameterisation of $\mathcal{L}_{m}(q, k)$. Given $z \in \mathcal{L}_{m}(q, k)$, there is a $t(z) \in\left[-\rho_{m} / 2+\right.$ $\left.\tau_{m}(q), 4 m-\rho_{m} / 2+\tau_{m}(q)\right] \cap \mathbb{Z}$ such that

$$
z=\left(\rho_{m}+2\left(t(z)-\tau_{m}(q)\right)-\varepsilon(z)+\frac{1}{2}\right) e_{1}-\beta_{m}(q, k) e_{2} .
$$

We also write, for $s \in \mathbb{Z}$,

$$
z_{q, k}(s)=\left(\rho_{m}+s-2 \tau_{m}(q)+\frac{1}{2}\right) e_{1}-\beta_{m}(q, k) e_{2},
$$

so that $\mathcal{L}_{m}(q, k)=\left\{z_{q, k}(s) ; s \in\left[-\rho_{m}+2 \tau_{m}(q)-1,8 m-\rho_{m}+2 \tau_{m}(q)\right] \cap \mathbb{Z}\right\}$.

Let

$$
\tilde{A}_{p, m}=\left\{s \in \mathbb{Z} ; 2\left[\alpha_{p}^{l} \lambda_{1}(2 m)^{1 / 3}\right]-1 \leq s<2\left[\alpha_{p}^{r} \lambda_{1}(2 m)^{1 / 3}\right]+1\right\} .
$$

The embedding of the interval $A_{p}$ as a discrete interval in $\mathcal{L}_{m}(q, k)$ is then given by

$$
I_{p, q, k}=\left\{z_{q, k}(s) ; s \in \tilde{A}_{p, m}\right\} .
$$


We define the random signed measure $\mu_{m}$ on $\left\{\beta_{1}, \ldots, \beta_{L_{1}}\right\} \times \mathbb{R}$ by

$$
\mu_{m}\left(\left\{\beta_{q}\right\} \times A_{p}\right)=\frac{1}{4 M} \sum_{k=1}^{M} \Delta h\left(I_{p, q, k}\right) \quad \text { for } 1 \leq p \leq L_{2}, 1 \leq q \leq L_{1} .
$$

The height changes between $a$-faces along a line are multiples of 4 . Intuitively, the factor 4 in the above normalisation ensures that we increase the height by 1 for each connected component of $a$ edges traversing the two boundaries, which in a sense are the paths describing the transition between the liquid and gas phases.

1.3. Main theorem. We now state the main theorem of this paper. Assume that $M=M(m) \rightarrow \infty$ as $m \rightarrow \infty$, but $M(m)(\log m)^{2} / m^{1 / 3} \rightarrow 0$ as $m \rightarrow \infty$, for example, we could take $M=(\log m)^{\gamma}$ for some $\gamma>0$.

THEOREM 1.1. The sequence of measures $\left\{\mu_{m}\right\}$ converges to $\mu_{\mathrm{Ai}}$ as $m$ tends to infinity in the sense that that there is an $R>0$ so that for all $\left|w_{p, q}\right| \leq R, 1 \leq$ $p \leq L_{2}, 1 \leq q \leq L_{1}$,

$$
\begin{aligned}
\lim _{m \rightarrow \infty} & \mathbb{E}\left[\exp \left(\sum_{p=1}^{L_{2}} \sum_{q=1}^{L_{1}} w_{p, q} \mu_{m}\left(\left\{\beta_{q}\right\} \times A_{p}\right)\right)\right] \\
= & \mathbb{E}\left[\exp \left(\sum_{p=1}^{L_{2}} \sum_{q=1}^{L_{1}} w_{p, q} \mu_{\mathrm{Ai}}\left(\left\{\beta_{q}\right\} \times A_{p}\right)\right)\right] .
\end{aligned}
$$

In the above equation, the expectation on the left-hand side is with respect to the two-periodic Aztec diamond measure and the right-hand side is with respect to the extended Airy point process.

1.4. Heuristic interpretation. The asymptotic formulas at the liquid-gas boundary for the inverse Kasteleyn matrix, described below, are computed in [9]. These formulas have a primary contribution from the full-plane gas phase inverse Kasteleyn matrix and a correction term given in terms of the extended Airy kernel. This means that when we consider correlations between dominoes that are relatively close they are essentially the same as in a pure gas phase. However, at longer distances the correction term becomes important since correlations in a pure gas phase decay exponentially. A heuristic description of the behaviour of the dominoes at the liquid-gas boundary is that the behaviour is primarily a gas phase but there is a family of random curves which have a much longer interaction scale than the gas phase objects. Although this is not quite an accurate description of the boundary, it naturally motivates the random measure $\mu_{m}$ defined in (1.11).

In this paper, we do not investigate whether there is a natural geometric curve that separates the liquid and gas regions. A candidate for such a path, the last treepath, is discussed in [9], Section 6, but there are other possible definitions. Such a 
path should converge to the Airy 2 process. The present work can be thought of as a crucial step in proving this by providing a specific averaging of the height function at the liquid-gas boundary which isolates the long-distance correlations. However, it does not give any direct information about the existence of a natural path that converges to the Airy2 process. We plan to investigate this in a future paper (work in progress).

1.5. Organisation. The rest of the paper is organised as follows: in Section 2, we introduce the particle description associated to the height function and the inverse Kasteleyn matrix. In Section 3, we state asymptotic formulas and results needed for the rest of the paper. The proof of Theorem 1.1 is given in Section 4. In Section 5, we give the proof of lemmas that are used in the proof of Theorem 1.1. Finally, in Section 6, we give the proof of the results stated in Section 3.

2. Inverse Kasteleyn matrix and the particle process. In this section, we introduce a particle process which will be used to prove (1.12). This particle process enables the direct use of determinantal point process machinery.

For the two-periodic Aztec diamond, there are two types of white vertices and two types of black vertices seen from the two possibilities of edge weights around each white and each black vertex. To distinguish between these types of vertices, we define for $i \in\{0,1\}$

$$
\mathrm{B}_{i}=\left\{\left(x_{1}, x_{2}\right) \in \mathrm{B}: x_{1}+x_{2} \bmod 4=2 i+1\right\}
$$

and

$$
\mathrm{W}_{i}=\left\{\left(x_{1}, x_{2}\right) \in \mathrm{W}: x_{1}+x_{2} \bmod 4=2 i+1\right\} .
$$

There are four different types of dimers having weight $a$ with $\left(\mathrm{W}_{i}, \mathrm{~B}_{j}\right)$ for $i, j \in$ $\{0,1\}$ and a further four types of dimers having weight 1 with $\left(\mathrm{W}_{i}, \mathrm{~B}_{j}\right)$ for $i, j \in$ $\{0,1\}$.

The Kasteleyn matrix for the two periodic Aztec diamond of size $n=4 m$ with parameters $a$ and $b$, denoted by $K_{a, b}$, is given by

$$
K_{a, b}(x, y)= \begin{cases}a(1-j)+b j & \text { if } y=x+e_{1}, x \in \mathrm{B}_{j}, \\ (a j+b(1-j)) \mathrm{i} & \text { if } y=x+e_{2}, x \in \mathrm{B}_{j}, \\ a j+b(1-j) & \text { if } y=x-e_{1}, x \in \mathrm{B}_{j}, \\ (a(1-j)+b j) \mathrm{i} & \text { if } y=x-e_{2}, x \in \mathrm{B}_{j}, \\ 0 & \text { if }(x, y) \text { is not an edge }\end{cases}
$$

where $\mathrm{i}^{2}=-1$ and $j \in\{0,1\}$. For the significance of the Kasteleyn matrix for random tiling models see, for example, [27].

Since the Aztec diamond graph is bipartite, meaning that there is a twocolouring of the vertices, from [24] the dimers of the two-periodic Aztec diamond 
form a determinantal point process. More explicitly, suppose that $E=\left\{e_{i}\right\}_{i=1}^{r}$ is a collection of distinct edges with $e_{i}=\left(\mathrm{b}_{i}, \mathrm{w}_{i}\right)$, where $\mathrm{b}_{i}$ and $\mathrm{w}_{i}$ denote black and white vertices.

THEOREM $2.1([23,24])$. The dimers form a determinantal point process on the edges of the Aztec diamond graph with correlation kernel L meaning that

$$
\mathbb{P}\left(\mathrm{e}_{1}, \ldots, \mathrm{e}_{r}\right)=\operatorname{det} L\left(\mathrm{e}_{i}, \mathrm{e}_{j}\right)_{1 \leq i, j \leq r},
$$

where

$$
L\left(\mathrm{e}_{i}, \mathrm{e}_{j}\right)=K_{a, b}\left(\mathrm{~b}_{i}, \mathrm{w}_{i}\right) K_{a, b}^{-1}\left(\mathrm{w}_{j}, \mathrm{~b}_{i}\right) .
$$

As mentioned in the Introduction, the derivation for the inverse Kasteleyn matrix, $K_{a, b}^{-1}$ for the two-periodic Aztec diamond is given in [10] and a simplification of this formula, which is amenable for asymptotic analysis, is given in [9]. For the purpose of this paper, we set $b=1$.

In order to prove (1.12), we want to write the expectation on the left-hand side as an expectation of a determinantal point process. For this, it is convenient to introduce a suitable particle process.

The space of possible particle positions is $\mathcal{L}_{m}$ given by (1.5). To a particle $z \in$ $\mathcal{L}_{m}$, we associate two vertices $x(z) \in \mathrm{W}$ and $y(z) \in \mathrm{B}$ and the edge $(y(z), x(z))$ between them. For $z \in \mathcal{L}_{m}$, and since each $z$ is incident to an $a$-face, we let

$$
\begin{aligned}
& x(z)=z-\frac{1}{2}(-1)^{\varepsilon(z)} e_{2}, \\
& y(z)=z+\frac{1}{2}(-1)^{\varepsilon(z)} e_{2} .
\end{aligned}
$$

This gives the particle to edge mapping

$$
\mathcal{L}_{m} \ni z \longleftrightarrow \quad(y(z), x(z)) \in \mathrm{B} \times \mathrm{W} .
$$

Using the definitions we see that $x(z) \in \mathrm{W}_{\varepsilon(z)}$ and $y(z) \in \mathrm{B}_{\varepsilon(z)}$; see Figure 6 .

From Theorem 2.1, we know that the dimers, that is, the covered edges, form a determinantal point process. Hence, the mapping (2.2) induces a determinantal point process on $\mathcal{L}_{m}$. There is a particle at $z \in \mathcal{L}_{m}$ if and only if the edge $(y(z), x(z))$ is covered by a dimer. The next proposition is an immediate consequence of Theorem 2.1 and the fact that $K_{a, 1}(y(z), x(z))=a \mathrm{i}$ for $z \in \mathcal{L}_{m}$.

Proposition 2.2. The particle process on $\mathcal{L}_{m}$ defined above is a determinantal point process with correlation kernel $\tilde{K}_{m}$ given by

$$
\tilde{\mathcal{K}}_{m}\left(z, z^{\prime}\right)=a \mathrm{i} K_{a, 1}^{-1}\left(x\left(z^{\prime}\right), y(z)\right)
$$

for $z, z^{\prime} \in \mathcal{L}_{m}$. 


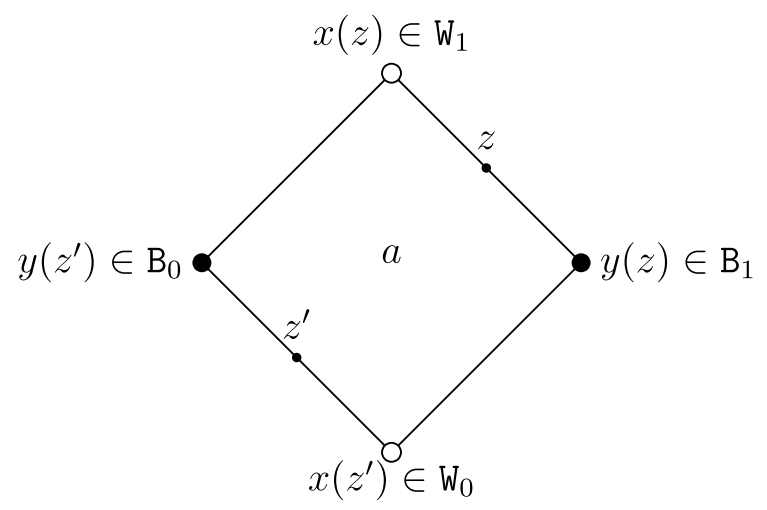

FIG. 6. An a-face on a discrete line with $\varepsilon\left(z^{\prime}\right)=0$ and $\varepsilon(z)=1, z, z^{\prime} \in \mathcal{L}_{m}$.

Recall the definitions of (1.10) and (1.11). Let $\left\{z_{i}\right\}$ denote the particle process on $\mathcal{L}_{m}$ as defined above, and let

$$
\mathbb{I}_{p, q, k}(z)= \begin{cases}1 & \text { if } z \in I_{p, q, k}, \\ 0 & \text { if } z \notin I_{p, q, k}\end{cases}
$$

be the indicator function for $I_{p, q, k}$. The change in the height function across the interval $I_{p, q, k}$ can be written in terms of the particle process, namely, we have the equation

$$
\Delta h\left(I_{p, q, k}\right)=4 \sum_{i}(-1)^{\varepsilon\left(z_{i}\right)} \mathbb{I}_{p, q, k}\left(z_{i}\right)
$$

where $\sum_{i}$ is the sum over all particles in the process. From the above equation and (1.11), we obtain

$$
\mu_{m}\left(\left\{\beta_{q}\right\} \times A_{p}\right)=\frac{1}{M} \sum_{k=1}^{M} \sum_{i}(-1)^{\varepsilon\left(z_{i}\right)} \mathbb{I}_{p, q, k}\left(z_{i}\right) .
$$

If we let

$$
\psi(z)=\sum_{k=1}^{M} \sum_{p=1}^{L_{2}} \sum_{q=1}^{L_{1}} w_{p, q}(-1)^{\varepsilon(z)} \mathbb{I}_{p, q, k}(z),
$$

we see that

$$
\sum_{p=1}^{L_{2}} \sum_{q=1}^{L_{1}} w_{p, q} \mu_{m}\left(\left\{\beta_{q}\right\} \times A_{p}\right)=\frac{1}{M} \sum_{i} \psi\left(z_{i}\right) .
$$


Since $\left\{z_{i}\right\}$ is a determinantal process on $\mathcal{L}_{m}$ with correlation kernel $\tilde{K}_{m}$, we immediately obtain

$$
\begin{aligned}
\mathbb{E}\left[\exp \left(\sum_{p=1}^{L_{2}} \sum_{q=1}^{L_{1}} w_{p, q} \mu_{m}\left(\left\{\beta_{q}\right\} \times A_{p}\right)\right)\right] \\
=\mathbb{E}\left[\exp \left(\frac{1}{M} \sum_{i} \psi\left(z_{i}\right)\right)\right] \\
=\mathbb{E}\left[\prod_{i}\left(1+\left(e^{\frac{1}{M} \psi\left(z_{i}\right)}-1\right)\right)\right] \\
=\operatorname{det}\left(\mathbb{I}+\left(e^{\frac{1}{M} \psi}-1\right) \tilde{\mathcal{K}}_{m}\right) .
\end{aligned}
$$

The matrix in the above determinant is indexed by entries of $\mathcal{L}_{m}$, which is a finite set. The above formula will be the basis of our asymptotic analysis which will lead to a proof of (1.12). To perform this asymptotic analysis, we need some asymptotic formulas which we state in the next section.

3. Asymptotic formulas. This section brings forward some of the key asymptotic results for the liquid-gas boundary from [9]. These results are refined specifically for the particle process introduced in Section 2 and the corresponding scaling associated to $\mathcal{L}_{m}$. The origin of these results is made explicit in Section 6 .

Let

$$
\tilde{c}\left(u_{1}, u_{2}\right)=2\left(1+a^{2}\right)+a\left(u_{1}+u_{1}^{-1}\right)\left(u_{2}+u_{2}^{-1}\right),
$$

which is related to the so-called characteristic polynomial for the dimer model [29]; see [9], (4.11), for an explanation. Write

$$
h\left(\varepsilon_{1}, \varepsilon_{2}\right)=\varepsilon_{1}\left(1-\varepsilon_{2}\right)+\varepsilon_{2}\left(1-\varepsilon_{1}\right) .
$$

We set

$$
\mathcal{C}=\frac{1}{\sqrt{2 c}}(1-\sqrt{1-2 c})
$$

REMARK 1. Note that the quantity $\mathcal{C}$ given above is exactly equal to the quantity $|G(\mathrm{i})|$ defined in [9], equation (2.6), that is, $|G(\mathrm{i})|=\mathcal{C}$. We have simplified the notation since only $|G(\mathrm{i})|$ appears in our computations here and the complete definition of $G$ along with its choice of branch cut is not necessary.

The full-plane gas phase inverse Kasteleyn matrix is given by

$$
\mathbb{K}_{1,1}^{-1}(x, y)
$$

$$
=-\frac{\mathrm{i}^{1+h\left(\varepsilon_{x}, \varepsilon_{y}\right)}}{(2 \pi \mathrm{i})^{2}} \int_{\Gamma_{1}} \frac{d u_{1}}{u_{1}} \int_{\Gamma_{1}} \frac{d u_{2}}{u_{2}} \frac{a^{\varepsilon_{y}} u_{2}^{1-h\left(\varepsilon_{x}, \varepsilon_{y}\right)}+a^{1-\varepsilon_{y}} u_{1} u_{2}^{h\left(\varepsilon_{x}, \varepsilon_{y}\right)}}{\tilde{c}\left(u_{1}, u_{2}\right) u_{1}^{\frac{x_{1}-y_{1}+1}{2}} u_{2}^{\frac{x_{2}-y_{2}+1}{2}}},
$$


where $x=\left(x_{1}, x_{2}\right) \in \mathrm{W}_{\varepsilon_{x}}$ and $y=\left(y_{1}, y_{2}\right) \in \mathrm{B}_{\varepsilon_{y}}$ with $\varepsilon_{x}, \varepsilon_{y} \in\{0,1\}$, and $\Gamma_{1}$ is the positively oriented unit circle; see [9], Section 4 , for details. For the rest of this paper, $\Gamma_{R}$ denotes a positively oriented circle of radius $R$ around the origin. From [9], it is natural to write

$$
K_{a, 1}^{-1}(x, y)=\mathbb{K}_{1,1}^{-1}(x, y)-\mathbb{K}_{\mathrm{A}}
$$

which defines $\mathbb{K}_{\mathrm{A}}$. The full expression for $\mathbb{K}_{\mathrm{A}}$ is complicated; see [9], Theorem 2.3. Its asymptotics is given in Section 6 . Since $K_{a, 1}(x(z), y(z))=a \mathrm{i}$, this leads us to define

$$
\begin{aligned}
& \tilde{\mathcal{K}}_{m, 0}\left(z, z^{\prime}\right)=a \mathrm{i} \mathbb{K}_{1,1}^{-1}\left(x\left(z^{\prime}\right), y(z)\right), \\
& \tilde{\mathcal{K}}_{m, 1}\left(z, z^{\prime}\right)=a \mathrm{i} \mathbb{K}_{\mathrm{A}}\left(x\left(z^{\prime}\right), y(z)\right)
\end{aligned}
$$

so that

$$
\tilde{\mathcal{K}}_{m}\left(z, z^{\prime}\right)=\sum_{\delta \in\{0,1\}}(-1)^{\delta} \tilde{\mathcal{K}}_{m, \delta}\left(z, z^{\prime}\right) .
$$

For $z \in \mathcal{L}_{m}(q, k)$, we define

$$
\begin{aligned}
& \gamma_{1}(z)=\frac{t(z)}{\lambda_{1}(2 m)^{1 / 3}} \beta_{q}-\frac{1}{3} \beta_{q}^{3}, \\
& \gamma_{2}(z)=\varepsilon(z)+\beta_{m}(q, k), \\
& \gamma_{3}(z)=2\left(t(z)-\tau_{m}(q)\right)+\beta_{m}(q, k) .
\end{aligned}
$$

We also introduce the relation

$$
\mathrm{g}_{\varepsilon_{1}, \varepsilon_{2}}= \begin{cases}\frac{\mathrm{i}\left(\sqrt{a^{2}+1}+a\right)}{1-a} & \text { if }\left(\varepsilon_{1}, \varepsilon_{2}\right)=(0,0), \\ \frac{\sqrt{a^{2}+1}+a-1}{\sqrt{2 a}(1-a)} & \text { if }\left(\varepsilon_{1}, \varepsilon_{2}\right)=(0,1), \\ -\frac{\sqrt{a^{2}+1}+a-1}{\sqrt{2 a}(1-a)} & \text { if }\left(\varepsilon_{1}, \varepsilon_{2}\right)=(1,0), \\ \frac{\mathrm{i}\left(\sqrt{a^{2}+1}-1\right)}{(1-a) a} & \text { if }\left(\varepsilon_{1}, \varepsilon_{2}\right)=(1,1) .\end{cases}
$$

Let $z, z^{\prime} \in \mathcal{L}_{m}$ and write $x\left(z^{\prime}\right)=\left(x_{1}\left(z^{\prime}\right), x_{2}\left(z^{\prime}\right)\right)$ and $y(z)=\left(y_{1}(z), y_{2}(z)\right)$. Motivated by the asymptotic results from [9] (compare [9], Theorem 2.7), we define for $\delta=0,1$,

$$
\begin{aligned}
\mathcal{K}_{m, \delta}\left(z, z^{\prime}\right)= & \frac{1}{\lambda_{1} c_{0} a \operatorname{ig}_{\varepsilon\left(z^{\prime}\right), \varepsilon(z)}} \mathrm{i}^{y_{1}(z)-x_{1}\left(z^{\prime}\right)-1} e^{\gamma_{1}\left(z^{\prime}\right)-\gamma_{1}(z)} \\
& \times \mathcal{C}^{\frac{1}{2}\left(2+x_{1}\left(z^{\prime}\right)-x_{2}\left(z^{\prime}\right)+y_{2}(z)-y_{1}(z)\right)} \tilde{\mathcal{K}}_{m, \delta}\left(z, z^{\prime}\right)
\end{aligned}
$$


and

$$
\mathcal{K}_{m}\left(z, z^{\prime}\right)=\sum_{\delta \in\{0,1\}}(-1)^{\delta} \mathcal{K}_{m, \delta}\left(z, z^{\prime}\right) .
$$

$\mathcal{K}_{m, \delta}\left(z, z^{\prime}\right)$ is the object that will have nice scaling limits and that we can control as $m \rightarrow \infty$; see Proposition 3.1 below. If $z \in \mathcal{L}_{m}(q, k)$ and $z^{\prime} \in \mathcal{L}_{m}\left(q^{\prime}, k^{\prime}\right)$, a computation using (1.7), (2.1) and (3.4) gives

$$
\frac{2+x_{1}\left(z^{\prime}\right)-x_{2}\left(z^{\prime}\right)+y_{2}(z)-y_{1}(z)}{2}=\gamma_{2}\left(z^{\prime}\right)-\gamma_{2}(z)+2-2 \varepsilon\left(z^{\prime}\right)
$$

and

$$
y_{1}(z)-x_{1}\left(z^{\prime}\right)-1=\gamma_{3}(z)-\gamma_{3}\left(z^{\prime}\right)+2 \varepsilon\left(z^{\prime}\right) .
$$

Applying these formulas in (3.5) and using the fact that $\lambda_{1} c_{0}=\frac{1}{2} \sqrt{1-2 c}$, we obtain

$$
\begin{aligned}
\tilde{\mathcal{K}}_{m, \delta}\left(z, z^{\prime}\right)= & \frac{a \mathrm{i}}{2} \sqrt{1-2 c} \mathrm{~g}_{\varepsilon\left(z^{\prime}\right), \varepsilon(z)} \mathcal{C}^{2 \varepsilon\left(z^{\prime}\right)-2}(-1)^{\varepsilon\left(z^{\prime}\right)} e^{\gamma_{1}\left(z^{\prime}\right)-\gamma_{1}(z)} \\
& \times \mathcal{C}^{\gamma_{2}(z)-\gamma_{2}\left(z^{\prime}\right)} \mathrm{i}^{\gamma_{3}\left(z^{\prime}\right)-\gamma_{3}(z)} \mathcal{K}_{m, \delta}\left(z, z^{\prime}\right) .
\end{aligned}
$$

From this, we see that

$$
\mathrm{K}_{m}\left(z, z^{\prime}\right)=\frac{a \mathrm{i}}{2} \sqrt{1-2 c} \mathrm{~g}_{\varepsilon\left(z^{\prime}\right), \varepsilon(z)} \mathcal{C}^{2 \varepsilon\left(z^{\prime}\right)-2}(-1)^{\varepsilon\left(z^{\prime}\right)} \mathcal{K}_{m}\left(z, z^{\prime}\right)
$$

is also a correlation kernel for the particle process on $\mathcal{L}_{m}$. See Section 6 for specific signposting of where these formulas come from.

The next proposition contains the asymptotic formulas and estimates that we will need in the proof of our main theorem. The proof will be given in Section 6 .

Proposition 3.1. Let $z \in \mathcal{L}_{m}(q, k), z \in \mathcal{L}_{m}\left(q^{\prime}, k^{\prime}\right)$ and write $t=t(z), t^{\prime}=$ $t\left(z^{\prime}\right)$. Consider $\mathcal{K}_{m, \delta}\left(z, z^{\prime}\right)$ defined by (3.5). The asymptotic formulas and estimates below are uniform as $m \rightarrow \infty$ for $|t|,\left|t^{\prime}\right| \leq C(2 m)^{1 / 3}$, for any fixed $C>0$ and $1 \leq k \leq M$.

1. For any $q, q^{\prime}$,

$$
\mathcal{K}_{m, 1}\left(z, z^{\prime}\right)=\frac{1}{\lambda_{1}(2 m)^{1 / 3}} \tilde{\mathcal{A}}\left(\beta_{q^{\prime}}, \frac{t^{\prime}}{\lambda_{1}(2 m)^{1 / 3}} ; \beta_{q}, \frac{t}{\lambda_{1}(2 m)^{1 / 3}}\right)(1+o(1)),
$$

where $\tilde{\mathcal{A}}$ is given in (1.1).

2. If $q \neq q^{\prime}$, then

$$
\mathcal{K}_{m, 0}\left(z, z^{\prime}\right)=\frac{1}{\lambda_{1}(2 m)^{1 / 3}} \phi_{\beta_{q^{\prime}}, \beta_{q}}\left(\frac{t^{\prime}}{\lambda_{1}(2 m)^{1 / 3}}, \frac{t}{\lambda_{1}(2 m)^{1 / 3}}\right)(1+o(1))
$$

where $c_{1}>0$ is a constant adn $\phi_{\beta_{q^{\prime}}, \beta_{q}}$ is given in (1.2). 
3. Assume that $q=q^{\prime}$ and $k>k^{\prime}$. Then there are constants $c_{1}, c_{2}, C>0$, so that

(a)

$$
\begin{aligned}
\mathcal{K}_{m, 0}\left(z, z^{\prime}\right)= & \frac{1}{\lambda_{1}(\log m)} \frac{1}{\sqrt{4 \pi\left(k-k^{\prime}\right)}} \\
& \times \exp \left(-\frac{1}{4\left(k-k^{\prime}\right)}\left(\frac{t^{\prime}-t}{\lambda_{1} \log m}\right)^{2}\right)(1+o(1))
\end{aligned}
$$

if $\left|t^{\prime}-t\right| \leq c_{2}\left(\left(k-k^{\prime}\right)(\log m)^{2}\right)^{7 / 12}$,

(b)

$$
\left|\mathcal{K}_{m, 0}\left(z, z^{\prime}\right)\right| \leq \frac{C}{(\log m) \sqrt{k-k^{\prime}}} \exp \left(-\frac{c_{1}}{\left(k-k^{\prime}\right)}\left(\frac{t^{\prime}-t}{\lambda_{1} \log m}\right)^{2}\right)
$$

if $c_{2}\left(\left(k-k^{\prime}\right)(\log m)^{2}\right)^{7 / 12} \leq\left|t^{\prime}-t\right| \leq \lambda_{2}\left(k-k^{\prime}\right)(\log m)^{2}$,

(c) and

$$
\left|\mathcal{K}_{m, 0}\left(z, z^{\prime}\right)\right| \leq C e^{-c_{1}\left(k-k^{\prime}\right)(\log m)^{2}}
$$

if $\left|t^{\prime}-t\right| \geq \lambda_{2}\left(k-k^{\prime}\right)(\log m)^{2}$.

4. Assume that $q=q^{\prime}$ and $k<k^{\prime}$. Then there are constants $c_{1}, C>0$ so that

$$
\left|\mathcal{K}_{m, 0}\left(z, z^{\prime}\right)\right| \leq C e^{-c_{1}\left(k^{\prime}-k\right)(\log m)^{2}} .
$$

5. Assume that $q=q^{\prime}$ and $k=k^{\prime}$. Then there are constants $c_{1}, C>0$ so that

$$
\left|\mathcal{K}_{m, 0}\left(z, z^{\prime}\right)\right| \leq C e^{-c_{1}\left|t^{\prime}-t\right|} .
$$

4. Proof of Theorem 1.1. In this section we give the proof of Theorem 1.1 relying on Proposition 3.1 and Lemmas 4.1 and 4.6 whose proofs are deferred to later in the paper. To prove Theorem 1.1, we analyze the right-hand side of (2.3) via its cumulant or trace expansion. Since $K_{m}$, given by (3.9), is also a correlation kernel for the particle process, we have

$$
\operatorname{det}\left(\mathbb{I}+\left(e^{\frac{1}{M} \psi}-1\right) \tilde{\mathcal{K}}_{m}\right)=\operatorname{det}\left(\mathbb{I}+\left(e^{\frac{1}{M} \psi}-1\right) \mathrm{K}_{m}\right) .
$$

For $\left|w_{p, q}\right| \leq R$ with $R$ sufficiently small, we have the expansion

$$
\begin{aligned}
& \log \operatorname{det}\left(\mathbb{I}+\left(e^{\frac{1}{M} \psi}-1\right) \mathrm{K}_{m}\right)_{\mathcal{L}_{m}} \\
& =\sum_{s=1}^{\infty} \frac{1}{M^{s}} \sum_{r=1}^{s} \frac{(-1)^{r+1}}{r} \sum_{\substack{\ell_{1}+\cdots+\ell_{r}=s \\
\ell_{1}, \ldots, \ell_{r} \geq 1}} \frac{1}{\ell_{1} ! \cdots \ell_{r} !} \operatorname{tr}\left(\psi^{\ell_{1}} \mathrm{~K}_{m} \cdots \psi^{\ell_{r}} \mathrm{~K}_{m}\right) .
\end{aligned}
$$

For a simple proof of this expansion see, for example, page 450 in [6]. Since $\mathcal{L}_{m}$ is finite we have a finite-dimensional operator, and the expansion is convergent if 
$R$ is small enough. Note that, a priori, $R$ could depend on $m$. It is a consequence of the proof below that we are able to choose $R$ independent of $m$.

Since all the discrete intervals $I_{p, q, k}$ have disjoint support,

$$
\psi(z)^{\ell}=\sum_{k=1}^{M} \sum_{p=1}^{L_{2}} \sum_{q=1}^{L_{1}} w_{p, q}^{\ell}(-1)^{\ell \varepsilon(z)} \mathbb{I}_{p, q, k}(z),
$$

for all $l \geq 1$. In what follows, we use the notation $\bar{j} \in S^{r}$ to denote the sum over all $j_{1}, \ldots, j_{r} \in S$ for some set $S$ and we assume the notation to be cyclic with respect to $r$, that is $j_{r+1}=j_{1}$. Also, we use the notation $[N]=\{1, \ldots, N\}$. Thus, we have from (4.2)

$$
\begin{aligned}
\operatorname{tr}\left(\psi^{\ell_{1}} \mathrm{~K}_{m} \cdots \psi^{\ell_{r}} \mathrm{~K}_{m}\right) & \\
= & \sum_{\bar{z} \in\left(\mathcal{L}_{m}\right)^{r}} \sum_{\bar{k} \in[M]^{r}} \sum_{\bar{p} \in\left[L_{2}\right]^{r}} \sum_{\bar{q} \in\left[L_{1}\right]^{r}} \prod_{i=1}^{r} w_{p_{i}, q_{i}}^{\ell_{i}}(-1)^{\ell_{i} \varepsilon\left(z_{i}\right)} \\
& \times \mathbb{I}_{p_{i}, q_{i}, k_{i}}\left(z_{i}\right) \mathrm{K}_{m}\left(z_{i}, z_{i+1}\right) \\
= & \sum_{\bar{\varepsilon} \in\{0,1\}^{r}} \sum_{\bar{k} \in[M]^{r}} \sum_{\bar{p} \in\left[L_{2}\right]^{r}} \sum_{\bar{q} \in\left[L_{1}\right]^{r}} \prod_{i=1}^{r} w_{p_{i}, q_{i}}^{\ell_{i}}(-1)^{\ell_{i} \varepsilon_{i}} \\
& \times \sum_{\bar{z} \in\left(\mathcal{L}_{m}\right)^{r}} \prod_{i=1}^{r} \mathbb{I}_{p_{i}, q_{i}, k_{i}}^{\varepsilon_{i}}\left(z_{i}\right) \mathrm{K}_{m}\left(z_{i}, z_{i+1}\right) .
\end{aligned}
$$

Here, $\mathbb{I}_{p, q, k}^{\varepsilon}$ is the indicator function on $\mathcal{L}_{m}$ for the set

$$
I_{p, q, k}^{\varepsilon}=\left\{z \in I_{p, q, k} ; \varepsilon(z)=\varepsilon\right\}
$$

for $\varepsilon \in\{0,1\}$. Write $\mathrm{K}_{m}=\sum_{\delta \in\{0,1\}}(-1)^{\delta} \mathrm{K}_{m, \delta}$, similar to (3.6), and plug it into (4.3) to get

$$
\begin{aligned}
\operatorname{tr}\left(\psi^{\ell_{1}} \mathrm{~K}_{m} \cdots \psi^{\ell_{r}} \mathrm{~K}_{m}\right) & \\
= & \sum_{\bar{\varepsilon}, \bar{\delta} \in\{0,1\}^{r}} \prod_{i=1}^{r}(-1)^{\ell_{i} \varepsilon_{i}} \sum_{\bar{k} \in[M]^{r}} \sum_{\bar{p} \in\left[L_{2}\right]^{r}} \sum_{\bar{q} \in\left[L_{1}\right]^{r}} \prod_{i=1}^{r} w_{p_{i}, q_{i}}^{\ell_{i}}(-1)^{\delta_{i}} \\
& \times \sum_{\bar{z} \in\left(\mathcal{L}_{m}\right)^{r}} \prod_{i=1}^{r} \mathbb{I}_{p_{i}, q_{i}, k_{i}}^{\varepsilon_{i}}\left(z_{i}\right) \mathrm{K}_{m, \delta_{i}}\left(z_{i}, z_{i+1}\right) .
\end{aligned}
$$

In order to carry out the asymptotic analysis, we will split this trace into four parts. Let

$$
D_{r}=\{0,1\}^{r} \times[M]^{r} \times\left[L_{2}\right]^{r} \times\left[L_{1}\right]^{r} .
$$


Define

$$
\begin{aligned}
D_{r, 0}= & \left\{(\bar{\delta}, \bar{k}, \bar{p}, \bar{q}) \in D_{r} ; \delta_{i}=0, k_{i}=k_{i+1}, p_{i}=p_{i+1}\right. \\
& \text { and } \left.q_{i}=q_{i+1}, 1 \leq i \leq r\right\}, \\
D_{r, 1}= & \left\{(\bar{\delta}, \bar{k}, \bar{p}, \bar{q}) \in D_{r} ; \delta_{i}=0, q_{i}=q_{i+1} \text { for } 1 \leq i \leq r\right. \\
& \text { and } \left.p_{i} \neq p_{i+1} \text { for some } i\right\}, \\
D_{r, 2}= & \left\{(\bar{\delta}, \bar{k}, \bar{p}, \bar{q}) \in D_{r} ; \delta_{i}=0, q_{i}=q_{i+1}, p_{i}=p_{i+1} \text { for } 1 \leq i \leq r\right. \\
& \text { and } \left.k_{i} \neq k_{i+1} \text { for some } i\right\}
\end{aligned}
$$

and

$$
D_{r, 3}=\left\{(\bar{\delta}, \bar{k}, \bar{p}, \bar{q}) \in D_{r} ; \delta_{i}=1 \text { or } q_{i} \neq q_{i+1} \text { for some } i\right\} .
$$

Then we have $D_{r}=D_{r, 0} \cup D_{r, 1} \cup D_{r, 2} \cup D_{r, 3}$. Introduce

$$
\begin{aligned}
T_{j}(m, r, \bar{l})= & \sum_{\bar{\varepsilon} \in\{0,1\}^{r}} \prod_{i=1}^{r}(-1)^{\ell_{i} \varepsilon_{i}} \sum_{(\bar{\delta}, \bar{k}, \bar{p}, \bar{q}) \in D_{r, j}} \prod_{i=1}^{r} w_{p_{i}, q_{i}}^{\ell_{i}}(-1)^{\delta_{i}} \\
& \times \sum_{\bar{z} \in\left(\mathcal{L}_{m}\right)^{r}} \prod_{i=1}^{r} \mathbb{1}_{p_{i}, q_{i}, k_{i}}^{\varepsilon_{i}}\left(z_{i}\right) \mathrm{K}_{m, \delta_{i}}\left(z_{i}, z_{i+1}\right),
\end{aligned}
$$

for $0 \leq j \leq 3$. Then, by (4.1) and (4.4) we have

$$
\log \operatorname{det}\left(\mathbb{I}+\left(e^{\frac{1}{M} \psi}-1\right) \mathrm{K}_{m}\right)=\sum_{j=0}^{3} U_{j}(m),
$$

where we define

$$
U_{j}(m)=\sum_{s=1}^{\infty} \frac{1}{M^{s}} \sum_{r=1}^{s} \frac{(-1)^{r+1}}{r} \sum_{\substack{\ell_{1}+\cdots+\ell_{r}=s \\ \ell_{1}, \ldots, \ell_{r} \geq 1}} \frac{T_{j}(m, r, \bar{\ell})}{\ell_{1} ! \cdots \ell_{r} !}
$$

Our goal is now to show that $U_{j}(m)$ tends to zero as $m$ tends infinity for $j=0,1,2$ and then to compute the limit of $U_{3}(m)$, which will give us what we want. The proof of $U_{0}(m)$ tends to zero as $m$ tends to infinity is rather involved and requires a separate argument. We formulate it as a lemma but postpone the proof until Section 5.

LEMMA 4.1. There is an $R>0$ such that $\lim _{m \rightarrow \infty} U_{0}(m)=0$ uniformly for $\left|w_{p, q}\right| \leq R$.

Recall (3.9) and define

$$
P(\bar{\varepsilon}, \bar{\ell})=\prod_{i=1}^{r} \frac{a \mathrm{i}}{2} \sqrt{1-2 c}(-1)^{\left(1+\ell_{i}\right) \varepsilon_{i}} \mathrm{~g}_{\varepsilon_{i}, \varepsilon_{i+1}} \mathcal{C}^{\varepsilon_{i}+\varepsilon_{i+1}-2} .
$$


Then we have

$$
\begin{aligned}
T_{j}(m, r, \bar{\ell})= & \sum_{\bar{\varepsilon} \in\{0,1\}^{r}} P(\bar{\varepsilon}, \bar{\ell}) \sum_{(\bar{\delta}, \bar{k}, \bar{p}, \bar{q}) \in D_{r, j}} \prod_{i=1}^{r} w_{p_{i}, q_{i}}^{\ell_{i}}(-1)^{\delta_{i}} \\
& \times \sum_{\bar{z} \in\left(\mathcal{L}_{m}\right)^{r}} \prod_{i=1}^{r} \mathbb{I}_{p_{i}, q_{i}, k_{i}}^{\varepsilon_{i}}\left(z_{i}\right) \mathcal{K}_{m, \delta_{i}}\left(z_{i}, z_{i+1}\right),
\end{aligned}
$$

for $j=1,2,3$.

Recall (1.7), (1.8), (1.9) and (1.10). Define

$$
z_{q, k}^{\varepsilon}(t)=\left(\rho_{m}+2\left(t-\tau_{m}(q)\right)-\varepsilon+\frac{1}{2}\right) e_{1}-\beta_{m}(q, k) e_{2}
$$

and

$$
A_{p, m}=\left\{t \in \mathbb{Z} ;\left[\alpha_{p}^{l} \lambda_{1}(2 m)^{1 / 3}\right] \leq t \leq\left[\alpha_{p}^{r} \lambda_{1}(2 m)^{1 / 3}\right]\right\}
$$

where we recall the notation $A_{p}=\left[\alpha_{p}^{l}, \alpha_{p}^{r}\right]$ for all $1 \leq p \leq L_{2}$. Then we can write

$$
I_{p, q, k}^{\varepsilon}=\left\{z_{q, k}^{\varepsilon}(t) ; t \in A_{p, m}\right\} .
$$

Hence, we can also write

$$
\begin{aligned}
S_{r}(\bar{\varepsilon}, \bar{\delta}, \bar{k}, \bar{p}, \bar{q}) & :=\sum_{\bar{z} \in\left(\mathcal{L}_{m}\right)^{r}} \prod_{i=1}^{r} \mathbb{I}_{p_{i}, q_{i}, k_{i}}^{\varepsilon_{i}}\left(z_{i}\right) \mathcal{K}_{m, \delta_{i}}\left(z_{i}, z_{i+1}\right) \\
& =\sum_{\bar{t} \in \mathbb{Z}^{r}} \prod_{i=1}^{r} \mathbb{I}_{A_{p_{i}, m}}\left(t_{i}\right) \mathcal{K}_{m, \delta_{i}}\left(z_{q_{i}, k_{i}}^{\varepsilon_{i}}\left(t_{i}\right), z_{q_{i+1}, k_{i+1}}^{\varepsilon_{i+1}}\left(t_{i+1}\right)\right) \\
& =\int_{\mathbb{R}^{r}} d^{r} \bar{t} \prod_{i=1}^{r} \mathbb{I}_{A_{p_{i}, m}}\left(\left[t_{i}\right]\right) \mathcal{K}_{m, \bar{\varepsilon}, \bar{\delta}, \bar{k}, \bar{q}}^{(i)}\left(t_{i}, t_{i+1}\right),
\end{aligned}
$$

where

$$
\mathcal{K}_{m, \bar{\varepsilon}, \bar{\delta}, \bar{k}, \bar{q}}^{(i)}\left(t, t^{\prime}\right)=\mathcal{K}_{m, \delta_{i}}\left(z_{q_{i}, k_{i}}^{\varepsilon_{i}}([t]), z_{q_{i+1}, k_{i+1}}^{\varepsilon_{i+1}}\left(\left[t^{\prime}\right]\right)\right) .
$$

With this notation, we see that

$$
\text { (4.10) } T_{j}(m, r, \bar{\ell})=\sum_{\bar{\varepsilon} \in\{0,1\}^{r}} P(\bar{\varepsilon}, \bar{\ell}) \sum_{(\bar{\delta}, \bar{k}, \bar{p}, \bar{q}) \in D_{r, j}} \prod_{i=1}^{r} w_{p_{i}, q_{i}}^{\ell_{i}}(-1)^{\delta_{i}} S_{r}(\bar{\varepsilon}, \bar{\delta}, \bar{k}, \bar{p}, \bar{q})
$$

for $j=1,2,3$.

LEMMA 4.2. There is an $R>0$ such that, for $j=1,2, \lim _{m \rightarrow \infty} U_{j}(m)=0$ uniformly in $\left|w_{p, q}\right| \leq R$. 
Proof. Consider $j=1$ so that $(\bar{\delta}, \bar{k}, \bar{p}, \bar{q}) \in D_{r, 1}$. There is an $i=i_{1}$ such that $p_{i_{1}} \neq p_{i_{1}+1}$ by the definition of $D_{r, 1}$. We have $\delta_{i}=0$ for all $i$. Hence, by statements (3) to (5) in Proposition 3.1, we have

$$
\left|\mathcal{K}_{m, \bar{\varepsilon}, \bar{\delta}, \bar{k}, \bar{q}}^{\left(i_{1}\right)}\left(t_{i_{1}}, t_{i_{1}+1}\right)\right| \leq C e^{-c_{1}(\log m)^{2}}
$$

since $\left|t_{i_{1}+1}-t_{i_{1}}\right| \geq C m^{1 / 3}$-note that the real estimate in the above inequality is actually less than or equal to $C e^{-c_{1} m^{1 / 3}}$ but we do not need this here. All the other $\mathcal{K}^{(i)}$ factors in the integrand in (4.9) can be estimated using statements (3) to (5) in Proposition 3.1; to make this argument very precise, we can use the same type of change of variables (4.11) below, we omit the details. From this, we see that

$$
\left|S_{r}(\bar{\varepsilon}, \bar{\delta}, \bar{k}, \bar{p}, \bar{q})\right| \leq C^{r} m^{2 / 3} e^{-c_{1}(\log m)^{2}} .
$$

Consequently, by (4.10), since $|P(\bar{\varepsilon}, \bar{\ell})| \leq C^{r}$,

$$
\left|T_{j}(m, r, \bar{\ell})\right| \leq C^{r} M^{r} R^{s} m^{2 / 3} e^{-c_{1}(\log m)^{2}} .
$$

We can use this estimate in (4.6) to see that

$$
\begin{aligned}
\left|U_{1}(m)\right| & \leq \sum_{s=1}^{\infty} \frac{R^{s}}{M^{s}} \sum_{r=1}^{s} \frac{1}{r} \sum_{\substack{\ell_{1}+\cdots+\ell_{r}=s \\
\ell_{1}, \ldots, \ell_{r} \geq 1}} \frac{(C M)^{r} m^{2 / 3} e^{-c_{1}(\log m)^{2}}}{\ell_{1} ! \cdots \ell_{r} !} \\
& \leq C m^{2 / 3} e^{-c_{1}(\log m)^{2}} \sum_{s=1}^{\infty}(C R)^{s} \leq C m^{2 / 3} e^{-c_{1}(\log m)^{2}}
\end{aligned}
$$

provided that $R$ is small enough. Here, we used the fact that

$$
\sum_{\substack{\ell_{1}+\cdots+\ell_{r}=s \\ \ell_{1}, \ldots, \ell_{r} \geq 1}} \frac{1}{\ell_{1} ! \cdots \ell_{r} !} \leq\left(\sum_{\ell=0}^{\infty} \frac{1}{\ell !}\right)^{r}=e^{r} .
$$

We next consider $j=2$ so that $(\bar{\delta}, \bar{k}, \bar{p}, \bar{q}) \in D_{r, 2}$. We cannot have $k_{i}>k_{i+1}$ for all $i$ since it violates the cyclic condition. Hence, when estimating the $\mathcal{K}^{(i)}$ in the integrand in (4.9), we have to use statement (4) in Proposition 3.1 at least once. We now proceed in exactly the same way as above to prove that $U_{2}(m) \rightarrow 0$ as $m \rightarrow \infty$.

It remains to consider $U_{3}(m)$. This means that we need to control $S_{r}(\bar{\varepsilon}, \bar{\delta}, \bar{k}$, $\bar{p}, \bar{q})$ in the case when $(\bar{\delta}, \bar{k}, \bar{p}, \bar{q}) \in D_{r, 3}$. There are two sub-cases, for a given $(\bar{\delta}, \bar{k}, \bar{p}, \bar{q}) \in D_{r, 3}$ :

1. if $\delta_{i}=1$ for some $i$, we define $i_{1}$, by $\delta_{1}=\cdots=\delta_{i_{1}-1}=0, \delta_{i_{1}}=1$,

2. if $\delta_{i}=0$ for all $i$, we define $i_{1}$ by $q_{1}=\cdots=q_{i_{1}} \neq q_{i_{1}+1}$. 
Such $i_{1}$ 's always exist by the definition of $D_{r, 3}$. Define $d_{i}, 1 \leq i \leq r$, by

$$
d_{i}= \begin{cases}\lambda_{1}(2 m)^{1 / 3} & \text { if } q_{i} \neq q_{i+1} \text { or } \delta_{i}=1, \\ (\log m) \lambda_{1} \sqrt{\left|k_{i+1}-k_{i}\right|} & \text { if } \delta_{i}=0, q_{i}=q_{i+1}, k_{i} \neq k_{i+1}, \\ 1 & \text { if } \delta_{i}=0, q_{i}=q_{i+1}, k_{i}=k_{i+1} .\end{cases}
$$

We now introduce new coordinates in (4.9) by

$$
\left\{\begin{array}{l}
\tau_{i_{1}}=t_{i_{1}} / d_{i_{1}}, \\
\tau_{i}=\left(t_{i+1}-t_{i}\right) / d_{i} \quad \text { if } i>i_{1},
\end{array}\right.
$$

recalling that the indices are cyclic. The inverse transformation is

$$
t_{i}=t_{i}(\bar{\tau})=\sum_{j=i_{1}}^{i} d_{j} \tau_{j}
$$

for $i_{1} \leq i<i_{1}+r$. After this change of variables, we obtain

$$
S_{r}(\bar{\varepsilon}, \bar{\delta}, \bar{k}, \bar{q})=\int_{\mathbb{R}^{r}} d^{r} \bar{\tau} \prod_{i=1}^{r} \mathbb{I}_{A_{p_{i}, m}}\left(\left[t_{i}(\bar{\tau})\right]\right) d_{i} \mathcal{K}_{m, \bar{\varepsilon}, \bar{\delta}, \bar{k}, \bar{q}}^{(i)}\left(t_{i}(\bar{\tau}), t_{i+1}(\bar{\tau})\right) .
$$

The next lemma gives a bound on $S_{r}$.

LEMMA 4.3. There is a constant $C>0$ such that

$$
\left|S_{r}(\bar{\varepsilon}, \bar{\delta}, \bar{k}, \bar{p}, \bar{q})\right| \leq C^{r}
$$

for all $(\bar{\varepsilon}, \bar{\delta}, \bar{k}, \bar{q}) \in D_{r, 3}$ and $\bar{\varepsilon} \in\{0,1\}^{r}$.

PROOF. If $\delta_{i}=1$, then statement (1) in Proposition 3.1 gives

$$
\left|d_{i} \mathcal{K}_{m, \bar{\varepsilon}, \bar{\delta}, \bar{k}, \bar{q}}^{(i)}\left(t_{i}(\bar{\tau}), t_{i+1}(\bar{\tau})\right)\right| \leq C\left|\tilde{\mathcal{A}}\left(\beta_{q_{i}}, \frac{t_{i}(\bar{\tau})}{\lambda_{1}(2 m)^{1 / 3}} ; \beta_{q_{i+1}}, \frac{t_{i+1}(\bar{\tau})}{\lambda_{1}(2 m)^{1 / 3}}\right)\right| .
$$

Similarly, if $\delta_{i}=0, q_{i} \neq q_{i+1}$, then

$$
\left|d_{i} \mathcal{K}_{m, \bar{\varepsilon}, \bar{\delta}, \bar{k}, \bar{q}}^{(i)}\left(t_{i}(\bar{\tau}), t_{i+1}(\bar{\tau})\right)\right| \leq C\left|\phi_{\beta_{q_{i}}, \beta_{q_{i+1}}}\left(\frac{t_{i}(\bar{\tau})}{\lambda_{1}(2 m)^{1 / 3}}, \frac{t_{i+1}(\bar{\tau})}{\lambda_{1}(2 m)^{1 / 3}}\right)\right|,
$$

by (2) in Proposition 3.1. Furthermore, we obtain the following estimates for $\delta_{i}=0$ and $q_{i}=q_{i+1}$ :

- If $k_{i}>k_{i+1}$ and $\left|\tau_{i}\right| \leq c_{2}\left(\left(k_{i}-k_{i+1}\right)(\log m)^{2}\right)^{1 / 2}$, then

$$
\left|d_{i} \mathcal{K}_{m, \bar{\varepsilon}, \bar{\delta}, \bar{k}, \bar{q}}^{(i)}\left(t_{i}(\bar{\tau}), t_{i+1}(\bar{\tau})\right)\right| \leq C e^{-c_{1}^{\prime} \tau_{i}^{2}}
$$

where $c_{1}^{\prime}>0$, which follows from statement (3)(a) in Proposition 3.1. 
- If $k_{i}<k_{i+1}$, or $k_{i}>k_{i+1}$ and $\left|\tau_{i}\right|>c_{2}\left(\left|k_{i}-k_{i+1}\right|(\log m)^{2}\right)^{1 / 2}$, then

$$
\left|d_{i} \mathcal{K}_{m, \bar{\varepsilon}, \bar{\delta}, \bar{k}, \bar{q}}^{(i)}\left(t_{i}(\bar{\tau}), t_{i+1}(\bar{\tau})\right)\right| \leq C e^{-c_{1}\left|k_{i}-k_{i+1}\right|(\log m)^{2}},
$$

which follows from statements (3)(a), (3)(b) and (4) in Proposition 3.1.

- If $k_{i}=k_{i+1}$, then

$$
\left|d_{i} \mathcal{K}_{m, \bar{\varepsilon}, \bar{\delta}, \bar{k}, \bar{q}}^{(i)}\left(t_{i}(\bar{\tau}), t_{i+1}(\bar{\tau})\right)\right| \leq C e^{-c_{1}\left|\tau_{i}\right|},
$$

which follows from statement (5) in Proposition 3.1.

If we use these estimates and the fact that $\left|t_{i}\right| \leq C m^{1 / 3}$ for all $1 \leq i \leq r$, we get the bound on $S_{r}$.

We can now prove that we have a uniform control of the series defining $U_{3}(m)$.

LEMMA 4.4. The series (4.6) defining $U_{3}(m)$ is uniformly convergent for $\left|w_{p, q}\right| \leq R$ if $R$ is sufficiently small.

PROOF. It follows from (4.6), (4.10) and the bound in Lemma 4.3 that

$$
\begin{aligned}
\left|U_{3}(m)\right| & \leq \sum_{s=1}^{\infty} \frac{1}{M^{s}} \sum_{r=1}^{s} \frac{1}{r} \sum_{\substack{\ell_{1}+\cdots+\ell_{r}=s \\
\ell_{1}, \ldots, \ell_{r} \geq 1}} \sum_{\bar{\varepsilon} \in\{0,1\}^{r}} \frac{|P(\bar{\varepsilon}, \bar{\ell})|}{\ell_{1} ! \ldots \ell_{r} !} \sum_{(\bar{\delta}, \bar{k}, \bar{p}, \bar{q}) \in D_{r, 3}} R^{s} C^{r} \\
& \leq \sum_{s=1}^{\infty} \frac{R^{s}}{M^{s}} \sum_{r=1}^{s}(C M)^{r}<\infty
\end{aligned}
$$

if $\left|w_{p, q}\right| \leq R$, and $R$ is sufficiently small.

Let

$$
D_{s, 3}^{*}=\left\{(\bar{\delta}, \bar{k}, \bar{p}, \bar{q}) \in D_{s, 3} ; k_{i} \neq k_{j} \text { for all } i \neq j\right\}
$$

and write

$$
Q(\bar{\varepsilon})=P(\bar{\varepsilon},(1, \ldots, 1))
$$

with the vector $(1, \ldots, 1)$ having length $s$. Define

$$
\begin{aligned}
U_{3}^{*}(m)= & \sum_{s=1}^{\infty} \frac{(-1)^{s+1}}{s M^{s}} \sum_{\bar{\varepsilon} \in\{0,1\}^{s}} Q(\bar{\varepsilon}) \\
& \times \sum_{(\bar{\delta}, \bar{k}, \bar{p}, \bar{q}) \in D_{s, 3}^{*}} \prod_{i=1}^{s}(-1)^{\delta_{i}} w_{p_{i}, q_{i}} S_{s}(\bar{\varepsilon}, \bar{\delta}, \bar{k}, \bar{p}, \bar{q}) .
\end{aligned}
$$


LEMmA 4.5. There is a constant $C>0$ so that for $\left|w_{p, q}\right| \leq R$, with $R$ sufficiently small,

$$
\left|U_{3}(m)-U_{3}^{*}(m)\right| \leq \frac{C}{M} .
$$

PROOF. The same argument as in the proof of the previous lemma shows that

$$
\begin{aligned}
& \mid \sum_{s=1}^{\infty} \frac{1}{M^{s}} \sum_{r=1}^{s-1} \frac{(-1)^{r+1}}{r} \sum_{\substack{\ell_{1}+\cdots+\ell_{r}=s \\
\ell_{1}, \ldots, \ell_{r} \geq 1}} \sum_{\bar{\varepsilon} \in\{0,1\}^{r}} \frac{P(\bar{\varepsilon}, \bar{\ell})}{\ell_{1} ! \ldots \ell_{r} !} \\
& \times \sum_{(\bar{\delta}, \bar{k}, \bar{p}, \bar{q}) \in D_{r, 3}} S_{r}(\bar{\varepsilon}, \bar{\delta}, \bar{k}, \bar{p}, \bar{q}) \mid \\
& \quad \leq \sum_{s=1}^{\infty} \frac{R^{s}}{M^{s}} \sum_{r=1}^{s-1} \frac{C^{r} M^{r}}{r} \leq \frac{C}{M} .
\end{aligned}
$$

If $r=s$, and $k_{i}=k_{j}$ for some $i, j$, then the number of elements in $D_{s, 3}$ is less than $C M^{s-1}$ and we use the same estimates as used in the proof of the previous lemma.

Since $M$ tends to infinity (slowly) as $m$ tends to infinity, we only have to consider $U_{3}^{*}(m)$.

Given $(\bar{\delta}, \bar{k}, \bar{p}, \bar{q}) \in D_{s, 3}^{*}$, we let $1 \leq j_{1}<\cdots<j_{r} \leq s$ be the indices $i$ where $\delta_{i}=1$, or $\delta_{i}=0$ and $q_{i} \neq q_{i+1}$. Let $\ell_{1}=j_{1}-j_{r}+s, \ell_{2}=j_{2}-j_{1}, \ldots, \ell_{r}=$ $j_{r}-j_{r-1}$. We see that $\ell_{i} \geq 1$ and $\ell_{1}+\cdots+\ell_{r}=s$. Also, $j_{r}=j_{1}+s-\ell_{1} \leq s$, which implies that $j_{1} \leq \ell_{1}$. Hence, given $\ell_{1}, \ldots, \ell_{r}$ with $\ell_{1}+\cdots+\ell_{r}=s, \ell_{i} \geq 1$ for all $1 \leq i \leq r$, and $j_{1}$ with $1 \leq j_{1} \leq \ell_{1}$, we can uniquely reconstruct $j_{1}, \ldots, j_{r}$.

Write $J=\left\{j_{1}, \ldots, j_{r}\right\}$ and $J^{\prime}=[s] \backslash J$. Then, using (4.9), we have

$$
\begin{aligned}
S_{s}(\bar{\varepsilon}, \bar{\delta}, \bar{k}, \bar{p}, \bar{q})= & \int_{\mathbb{R}^{s}} d^{s} \bar{\tau} \prod_{i=1}^{s} \mathbb{I}_{A_{p_{i}, m}}\left(\left[t_{i}(\bar{\tau})\right]\right) \prod_{i \in J} d_{i} \mathcal{K}_{m, \bar{\varepsilon}, \bar{\delta}, \bar{k}, \bar{q}}^{(i)}\left(t_{i}(\bar{\tau}), t_{i+1}(\bar{\tau})\right) \\
& \times \prod_{i \notin J} d_{i} \mathcal{K}_{m, \bar{\varepsilon}, \bar{\delta}, \bar{k}, \bar{q}}^{(i)}\left(t_{i}(\bar{\tau}), t_{i+1}(\bar{\tau})\right) .
\end{aligned}
$$

Note that $\left[t_{i}(\bar{\tau})\right] \in A_{p_{i}, m}$ means that

$$
\left[\alpha_{p_{i}}^{l} \lambda_{1}(2 m)^{1 / 3}\right] \leq t_{i}(\tau) \leq\left[\alpha_{p_{i}}^{r} \lambda_{1}(2 m)^{1 / 3}\right] .
$$

Dropping the integer parts gives a negligible error and this is equivalent to $t_{i}(\tau) / \lambda_{1}(2 m)^{1 / 3} \in A_{p_{i}}$, where $A_{p_{i}}=\left[\alpha_{p_{i}}^{l}, \alpha_{p_{i}}^{r}\right]$. By statement (3) in Proposition 3.1, for $i \in J^{\prime}$ and $\left|\tau_{i}\right| \leq c_{2}(\log m)^{1 / 6}$,

$$
d_{i} \mathcal{K}_{m, \bar{\varepsilon}, \bar{\delta}, \bar{k}, \bar{q}}^{(i)}\left(t_{i}(\bar{\tau}), t_{i+1}(\bar{\tau})\right)=\frac{1}{\sqrt{4 \pi}} e^{-\frac{\tau_{i}^{2}}{4}}(1+o(1)) \mathbb{I}_{k_{i}>k_{i+1}}
$$


as $m \rightarrow \infty$. Write

$$
B_{0}\left(\beta, \xi ; \beta^{\prime}, \xi^{\prime}\right)=\phi_{\beta, \beta^{\prime}}\left(\xi, \xi^{\prime}\right)
$$

and

$$
B_{1}\left(\beta, \xi ; \beta^{\prime}, \xi^{\prime}\right)=\tilde{\mathcal{A}}\left(\beta, \xi ; \beta^{\prime}, \xi^{\prime}\right)
$$

Then, for $i \in J$ and $\left|t_{i}(\bar{\tau})\right|,\left|t_{i+1}(\bar{\tau})\right| \leq C m^{1 / 3}$,

$$
d_{i} \mathcal{K}_{m, \bar{\varepsilon}, \bar{\delta}, \bar{k}, \bar{q}}^{(i)}\left(t_{i}(\bar{\tau}), t_{i+1}(\bar{\tau})\right)=B_{\delta_{i}}\left(\beta_{q_{i}}, \frac{t_{i}(\bar{\tau})}{\lambda_{1}(2 m)^{1 / 3}} ; \beta_{q_{i+1}}, \frac{t_{i+1}(\bar{\tau})}{\lambda_{1}(2 m)^{1 / 3}}\right)(1+o(1))
$$

as $m \rightarrow \infty$. Note that

$$
\lim _{m \rightarrow \infty} \frac{t_{i}(\bar{\tau})}{\lambda_{1}(2 m)^{1 / 3}}=\lim _{m \rightarrow \infty} \sum_{j=i_{1}}^{i} \frac{d_{j}}{\lambda_{1}(2 m)^{1 / 3}} \tau_{j}=\sum_{\substack{j=i_{1} \\ j \in J}}^{i} \tau_{j}
$$

for $i_{1} \leq i<i_{1}+s$.

It follows from the above asymptotic formulas and the estimates in Proposition 3.1 that

$$
\begin{aligned}
& \lim _{m \rightarrow \infty} S_{S}(\bar{\varepsilon}, \bar{\delta}, \bar{k}, \bar{p}, \bar{q}) \\
&=\int_{\mathbb{R}^{r}} \prod_{j \in J} d \tau_{j} \prod_{i=1}^{s} \mathbb{I}_{A_{p_{i}}}\left(\sum_{j=i_{1}, j \in J}^{i} \tau_{j}\right) \\
& \times \prod_{i \in J} B_{\delta_{i}}\left(\beta_{q_{i}}, \sum_{j=i_{1}, j \in J}^{i} \tau_{j} ; \beta_{q_{i+1}}, \sum_{j=i_{1}, j \in J}^{i+1} \tau_{j}\right) \\
& \times \prod_{i \in J^{\prime}} \mathbb{I}_{k_{i}>k_{i+1}} \frac{1}{\sqrt{4 \pi}} \int_{\mathbb{R}} d \tau_{i} e^{-\frac{\tau_{i}^{2}}{4}} s \\
&= \prod_{i \in J^{\prime}} \mathbb{I}_{k_{i}>k_{i+1}} \int_{\mathbb{R}^{r}} \prod_{j \in J} d \tau_{j} \prod_{i=1}^{s} \mathbb{I}_{A_{p_{i}}}\left(\sum_{j=i_{1}, j \in J}^{i} \tau_{j}\right) \\
& \times \prod_{i \in J} B_{\delta_{i}}\left(\beta_{q_{i}}, \sum_{j=i_{1}, j \in J}^{i} \tau_{j} ; \beta_{q_{i+1}}, \sum_{j=i_{1}, j \in J}^{i+1} \tau_{j}\right) .
\end{aligned}
$$

Note that a nonzero right-hand side in (4.16) requires $p_{i}=p_{j_{\alpha}}$ for $j_{\alpha} \leq i<j_{\alpha+1}$ since otherwise

$$
\prod_{i=1}^{s} \mathbb{I}_{A_{p_{i}}}\left(\sum_{j=i_{1}, j \in J}^{i} \tau_{j}\right)=0
$$


By the definition of $j_{\alpha}$, we have that $q_{i}=q_{j_{\alpha}}$ for $j_{\alpha} \leq i<j_{\alpha+1}$. Note that the limit in (4.16) does not depend on $\varepsilon$. We have, for fixed $\delta, q$, which determine $J$ and $J^{\prime}$, that

$$
\lim _{m \rightarrow \infty} \frac{1}{M^{s}} \sum_{\bar{k} \in[M]^{s}} \prod_{i \in J^{\prime}} \mathbb{I}_{k_{i}>k_{i+1}}=\frac{1}{\ell_{1} ! \cdots \ell_{r} !} .
$$

Thus, after an analogous change of variables to (4.11), we get

$$
\begin{aligned}
\lim _{m \rightarrow \infty} & \frac{1}{M^{s}} \sum_{\substack{(\bar{\delta}, \bar{k}, \bar{p}, \bar{q}) \in D_{s, 3}^{*} \\
=}} \prod_{i=1}^{s}(-1)^{\delta_{i}} w_{p_{i}, q_{i}} S_{s}(\bar{\varepsilon}, \bar{\delta}, \bar{k}, \bar{p}, \bar{q}) \\
= & \sum_{r=1}^{s} \sum_{\ell_{1}+\ldots+\ell_{r}=s} \frac{\ell_{1}(-1)^{r}}{\ell_{1} ! \ldots \ell_{r} !} \sum_{\bar{p} \in\left[L_{2}\right]^{r}} \sum_{\bar{q} \in\left[L_{1}\right]^{r}} \sum_{\bar{\delta} \in\{0,1\}^{r}} \prod_{i=1}^{r}(-1)^{1+\delta_{i}} w_{p_{i}, q_{i}}^{\ell_{i}} \\
& \times \int_{\mathbb{R}^{r}} d^{r} \bar{t} \prod_{i=1}^{r} \mathbb{I}_{p_{p_{i}}}\left(t_{i}\right) B_{\delta_{i}}\left(\beta_{q_{i}}, t_{i} ; \beta_{q_{i+1}}, t_{i+1}\right),
\end{aligned}
$$

where the $\ell_{1}$ factor comes from the $\ell_{1}$ possible choices of $j_{1}$ as discussed above. By symmetry, we see that we can replace

$$
\sum_{\substack{\ell_{1}+\cdots+\ell_{r}=s \\ \ell_{1}, \ldots, \ell_{r} \geq 1}} \frac{\ell_{1}(-1)^{r}}{\ell_{1} ! \cdots \ell_{r} !}
$$

on the right-hand side of (4.17) by

$$
\frac{1}{r} \sum_{\substack{\ell_{1}+\cdots+\ell_{r}=s \\ \ell_{1}, \ldots, \ell_{r} \geq 1}} \frac{\left(\ell_{1}+\cdots+\ell_{r}\right)(-1)^{r}}{\ell_{1} ! \cdots \ell_{r} !}=\frac{(-1)^{r}}{r} \sum_{\substack{\ell_{1}+\cdots+\ell_{r}=s \\ \ell_{1}, \ldots, \ell_{r} \geq 1}} \frac{s}{\ell_{1} ! \ldots \ell_{r} !} .
$$

Thus, we find that

$$
\begin{aligned}
\lim _{m \rightarrow \infty} & \frac{1}{M^{s}} \sum_{(\bar{\delta}, \bar{k}, \bar{p}, \bar{q}) \in D_{s, 3}^{*}} \prod_{i=1}^{s}(-1)^{\delta_{i}} w_{p_{i}, q_{i}} S_{s}(\bar{\varepsilon}, \bar{\delta}, \bar{k}, \bar{p}, \bar{q}) \\
= & \sum_{r=1}^{s} \frac{(-1)^{r}}{r} \sum_{\substack{\ell_{1}+\cdots+\ell_{r}=s \\
\ell_{1}, \ldots, \ell_{r} \geq 1}} \frac{s}{\ell_{1} ! \ldots \ell_{r} !} \sum_{\bar{p} \in\left[L_{2}\right]^{r}} \sum_{\bar{q} \in\left[L_{1}\right]^{r}} \prod_{i=1}^{r} w_{p_{i}, q_{i}}^{\ell_{i}} \\
& \times \int_{\mathbb{R}^{r}} d^{r} \bar{t} \prod_{i=1}^{r} \mathbb{I}_{A_{p_{i}}}\left(t_{i}\right) \mathcal{A}\left(\beta_{q_{i}}, t_{i} ; \beta_{q_{i+1}}, t_{i+1}\right),
\end{aligned}
$$

since $\mathcal{A}=-B_{0}+B_{1}$ from (1.3), (4.14) and (4.15). In order to get the limit of $U_{3}^{*}(m)$ in (4.13), we need the following lemma, which we will prove in Section 5. 
LEMMA 4.6. We have that

$$
\sum_{\bar{\varepsilon} \in\{0,1\}^{s}} Q(\bar{\varepsilon})=(-1)^{s} .
$$

Thus, using the estimate in Lemma 4.3, we see that, provided $\left|w_{p, q}\right| \leq R$ with $R$ sufficiently small, we can take the limit in (4.13) and get

$$
\begin{aligned}
\lim _{m \rightarrow \infty} U_{3}^{*}(m)= & \sum_{s=1}^{\infty} \sum_{r=1}^{s} \frac{(-1)^{r+1}}{r} \sum_{\substack{\ell_{1}+\cdots+\ell_{r}=s \\
\ell_{1}, \ldots, \ell_{r} \geq 1}} \frac{1}{\ell_{1} ! \ldots \ell_{r} !} \sum_{\bar{p} \in\left[L_{2}\right]^{r}} \sum_{\bar{q} \in\left[L_{1}\right]^{r}} \prod_{i=1}^{r} w_{p_{i}, q_{i}}^{\ell_{i}} \\
& \times \int_{\mathbb{R}^{r}} d^{r} \bar{t} \prod_{i=1}^{r} \mathbb{I}_{A_{p_{i}}}\left(t_{i}\right) \mathcal{A}\left(\beta_{q_{i}}, t_{i} ; \beta_{q_{i+1}}, t_{i+1}\right) \\
= & \log \operatorname{det}\left(\mathbb{I}+\left(e^{\Psi}-1\right)\right)_{L^{2}\left(\left\{\beta_{1}, \ldots, \beta_{L_{1}}\right\} \times \mathbb{R}\right)},
\end{aligned}
$$

where $\Psi(x)=\sum_{p=1}^{L_{2}} \sum_{q=1}^{L_{1}} w_{p, q} \mathbb{I}_{\left\{\beta_{q}\right\} \times A_{p}}(x)$ as defined in (1.4) for $x \in\left\{\beta_{1}, \ldots\right.$, $\left.\beta_{q}\right\} \times \mathbb{R}$. This completes the proof of the theorem.

5. Proofs of Lemmas 4.1 and 4.6. In this section, we will give the proof of Lemma 4.1 followed by the proof of Lemma 4.6. These were both stated without proof in Section 4.

Before giving the proof of Lemma 4.1, we recall notation and give some preliminaries. As in Section 4, we assume that the notation is cyclic, that is, $z_{r+1}=z_{1}$ in all products of size $r$. Note that since $\mathrm{K}_{m, 0}$ is related to $\tilde{\mathcal{K}}_{m, 0}=a \mathrm{i} \mathbb{K}_{1,1}^{-1}$ by a conjugation [see (3.3), (3.8) and (3.9)], we have

$$
\prod_{i=1}^{r} \mathrm{~K}_{m, 0}\left(z_{i}, z_{i+1}\right)=\prod_{i=1}^{r} a \mathrm{i} \mathbb{K}_{1,1}^{-1}\left(x\left(z_{i+1}\right), y\left(z_{i}\right)\right) .
$$

Let $t=t(z), t^{\prime}=t\left(z^{\prime}\right), \varepsilon=\varepsilon(z)$ and $\varepsilon^{\prime}=\varepsilon\left(z^{\prime}\right)$, where $z, z^{\prime} \in \mathcal{L}_{m}(q, k)$. From (3.2), we see that

$$
\begin{aligned}
\mathbb{K}_{1,1}^{-1}\left(x\left(z^{\prime}\right), y(z)\right)= & -\frac{\mathrm{i}^{1+h\left(\varepsilon_{1}, \varepsilon_{2}\right)}}{(2 \pi \mathrm{i})^{2}} \int_{\Gamma_{1}} \frac{d u_{1}}{u_{1}} \int_{\Gamma_{1}} \frac{d u_{2}}{u_{2}} \\
& \times \frac{a^{\varepsilon} u_{2}^{1-h\left(\varepsilon, \varepsilon^{\prime}\right)}+a^{1-\varepsilon} u_{1} u_{2}^{h\left(\varepsilon, \varepsilon^{\prime}\right)}}{\tilde{c}\left(u_{1}, u_{2}\right) u_{1}^{\frac{x_{1}\left(z^{\prime}\right)-y_{1}(z)+1}{2}} u_{2}^{\frac{x_{2}\left(z^{\prime}\right)-y_{2}(z)+1}{2}}} .
\end{aligned}
$$

Now, we have $x_{2}\left(z^{\prime}\right)-y_{2}(z)=2\left(t^{\prime}-t\right)-1+2 \varepsilon$ and $x_{1}\left(z^{\prime}\right)-y_{1}(z)=2\left(t^{\prime}-t\right)+$ $1-2 \varepsilon^{\prime}$ by (1.7) and (2.1). Define

$$
\begin{aligned}
\mathcal{G}_{\varepsilon, \varepsilon^{\prime}}(t)= & \frac{a \mathrm{i}^{h\left(\varepsilon, \varepsilon^{\prime}\right)}}{(2 \pi \mathrm{i})^{2}} \int_{\Gamma_{1}} \frac{d u_{1}}{u_{1}} \\
& \times \int_{\Gamma_{1}} \frac{d u_{2}}{u_{2}} \frac{a^{\varepsilon} u_{1}^{-1+\varepsilon+\varepsilon^{\prime}} u_{2}^{1-h\left(\varepsilon, \varepsilon^{\prime}\right)}+a^{1-\varepsilon} u_{1}^{\varepsilon+\varepsilon^{\prime}} u_{2}^{h\left(\varepsilon, \varepsilon^{\prime}\right)}}{\tilde{c}\left(u_{1}, u_{2}\right)\left(u_{1} u_{2}\right)^{t+\varepsilon}} .
\end{aligned}
$$


It follows that

$$
a \mathrm{i} \mathbb{K}_{1,1}^{-1}\left(x\left(z^{\prime}\right), y(z)\right)=\mathcal{G}_{\varepsilon, \varepsilon^{\prime}}\left(t^{\prime}-t\right)
$$

and consequently

$$
\prod_{i=1}^{r} \mathrm{~K}_{m, 0}\left(z_{i}, z_{i+1}\right)=\prod_{i=1}^{r} \mathcal{G}_{\varepsilon_{i}, \varepsilon_{i+1}}\left(t_{i+1}-t_{i}\right)
$$

if $z_{i} \in \mathcal{L}_{m}(q, k), \varepsilon_{i}=\varepsilon\left(z_{i}\right)$ and $t_{i}=t\left(z_{i}\right)$ for $1 \leq i \leq r$. By making the change of variables $u_{1}=u, u_{2}=\omega / u$ in (5.2), we obtain

$$
\mathcal{G}_{\varepsilon, \varepsilon^{\prime}}(t)=\frac{a \mathrm{i}^{h\left(\varepsilon, \varepsilon^{\prime}\right)}}{2 \pi \mathrm{i}} \int_{\Gamma_{1}} \frac{f_{\varepsilon, \varepsilon^{\prime}}(\omega)}{\omega^{t}} \frac{d \omega}{\omega}
$$

where

$$
f_{\varepsilon, \varepsilon^{\prime}}(\omega)=\frac{1}{2 \pi \mathrm{i}} \int_{\Gamma_{1}} \frac{d u}{u} \frac{a^{\varepsilon} u^{-2(1-\varepsilon)\left(1-\varepsilon^{\prime}\right)} \omega^{1-\varepsilon-h\left(\varepsilon, \varepsilon^{\prime}\right)}+a^{1-\varepsilon} u^{2 \varepsilon \varepsilon^{\prime}} \omega^{h\left(\varepsilon, \varepsilon^{\prime}\right)-\varepsilon}}{\tilde{c}(u, \omega / u)}
$$

We have the following lemma.

LEMMA 5.1. Let $f_{\varepsilon, \varepsilon^{\prime}}(\omega)$ be defined in (5.5). Then we have the relations

$$
f_{0,0}(\omega)=f_{1,1}(\omega)
$$

and

$$
a f_{0,0}(\omega)-a^{2}\left(f_{0,0}(\omega)^{2}+f_{0,1}(\omega) f_{1,0}(\omega)\right)=0
$$

ProOF. From (3.1), we have

$$
\begin{aligned}
\tilde{c}(\sqrt{u}, \omega / \sqrt{u}) & =\frac{a}{u \omega}\left(u^{2}+\left(1+2(a+1 / a) \omega+\omega^{2}\right) u+\omega^{2}\right) \\
& =\frac{a}{u \omega}\left(u-r_{1}(\omega)\right)\left(u-r_{2}(\omega)\right) .
\end{aligned}
$$

The term in the parenthesis on the right-hand side of the first line of the above equation is a quadratic in $u$ and the second line gives the factorisation into two roots, $r_{1}(\omega)$ and $r_{2}(\omega)$. We have that $r_{1}(\omega) r_{2}(\omega)=\omega^{2}$ and so for $\omega \in \mathbb{T}$, we choose $\left|r_{1}(\omega)\right|<1$ and $\left|r_{2}(\omega)\right|>1$.

Making the change of variables $u \mapsto \sqrt{u}$ for $f_{\varepsilon, \varepsilon^{\prime}}(\omega)$, defined in (5.5), gives

$$
\begin{aligned}
f_{\varepsilon, \varepsilon^{\prime}}(z) & =\frac{1}{2 \pi \mathrm{i}} \int_{\Gamma_{1}} \frac{d u}{u} \frac{a^{\varepsilon} \omega^{1-\varepsilon-h\left(\varepsilon, \varepsilon^{\prime}\right)} u^{-(1-\varepsilon)\left(1-\varepsilon^{\prime}\right)}+a^{1-\varepsilon} u^{\varepsilon \varepsilon^{\prime}} \omega^{h\left(\varepsilon, \varepsilon^{\prime}\right)-\varepsilon}}{\tilde{c}(\sqrt{u}, \omega / \sqrt{u})} \\
& =\frac{1}{2 \pi \mathrm{i}} \int_{\Gamma_{1}} d u \frac{a^{\varepsilon-1} \omega^{2-h\left(\varepsilon, \varepsilon^{\prime}\right)-\varepsilon} u^{-(1-\varepsilon)\left(1-\varepsilon^{\prime}\right)}+a^{-\varepsilon} u^{\varepsilon \varepsilon^{\prime}} \omega^{1+h\left(\varepsilon, \varepsilon^{\prime}\right)-\varepsilon}}{\left(u-r_{1}(\omega)\right)\left(u-r_{2}(\omega)\right)} .
\end{aligned}
$$


In the above integrand for $\left(\varepsilon, \varepsilon^{\prime}\right) \neq(0,0)$, then $(1-\varepsilon)\left(1-\varepsilon^{\prime}\right)=0$ which means that there is only residue at $u=r_{1}(z)$. This is easily computed and gives

$$
\frac{a^{\varepsilon-1} \omega^{2-h\left(\varepsilon, \varepsilon^{\prime}\right)-\varepsilon}+a^{-\varepsilon} r_{1}(\omega)^{\varepsilon \varepsilon^{\prime}} \omega^{1+h\left(\varepsilon, \varepsilon^{\prime}\right)-\varepsilon}}{r_{1}(\omega)-r_{2}(\omega)} .
$$

For $\left(\varepsilon, \varepsilon^{\prime}\right)=(0,0)$, there are residues at $u=r_{1}(\omega)$ and $u=0$ which give

$$
\begin{aligned}
& \frac{a^{-1} \omega^{2} r_{1}(\omega)^{-1}+\omega}{r_{1}(\omega)-r_{2}(\omega)}+\frac{a^{-1} \omega^{2}}{r_{1}(\omega) r_{2}(\omega)} \\
& \quad=\frac{r_{1}(\omega) r_{2}(\omega)\left(a^{-1} \omega^{2} r_{1}(\omega)^{-1}+\omega\right)+a^{-1} \omega^{2}\left(r_{1}(\omega)-r_{2}(\omega)\right)}{r_{1}(\omega) r_{2}(\omega)\left(r_{1}(\omega)-r_{2}(\omega)\right)} \\
& \quad=\frac{a^{-1} r_{1}(\omega)+\omega}{r_{1}(\omega)-r_{2}(\omega)}
\end{aligned}
$$

where we have used $r_{1}(\omega) r_{2}(\omega)=\omega^{2}$. We have arrived at

$$
\begin{aligned}
f_{\varepsilon, \varepsilon^{\prime}}(\omega) & \\
= & \left(a^{\varepsilon-1} r_{1}(\omega)^{(1-\varepsilon)\left(1-\varepsilon^{\prime}\right)} \omega^{2-2(1-\varepsilon)\left(1-\varepsilon^{\prime}\right)-h\left(\varepsilon, \varepsilon^{\prime}\right)-\varepsilon}\right. \\
& \left.+a^{-\varepsilon} \omega^{1+h\left(\varepsilon, \varepsilon^{\prime}\right)-\varepsilon} r_{1}(\omega)^{\varepsilon \varepsilon^{\prime}}\right) /\left(r_{1}(\omega)-r_{2}(\omega)\right) .
\end{aligned}
$$

Using the above equation, the first equation in Lemma 5.1 immediately follows. For the second in equation in Lemma 5.1, using (5.6) we have

$$
\begin{aligned}
a f_{0,0}(\omega) & -a^{2}\left(f_{0,0}(\omega)^{2}+f_{0,1}(\omega) f_{1,0}(\omega)\right) \\
= & a \frac{a^{-1} r_{1}(\omega)+\omega}{r_{1}(\omega)-r_{2}(\omega)} \\
& -\frac{a^{2}}{\left(r_{1}(\omega)-r_{2}(\omega)\right)^{2}}\left(\left(a^{-1} r_{1}(\omega)+\omega\right)^{2}+\left(a^{-1} \omega+\omega^{2}\right)\left(1+a^{-1} \omega\right)\right) \\
= & -\frac{2 a^{2} \omega^{2}+a r_{1}(\omega) \omega+a r_{2}(\omega) \omega+a \omega^{3}+a \omega+r_{1}(\omega) r_{2}(\omega)+\omega^{2}}{\left(r_{1}(\omega)-r_{2}(\omega)\right)^{2}} \\
= & -\frac{\omega\left(2 a^{2} \omega+a\left(r_{1}(\omega)+r_{2}(\omega)+\omega^{2}+1\right)+2 \omega\right)}{\left(r_{1}(\omega)-r_{2}(\omega)\right)^{2}}=0,
\end{aligned}
$$

where we have used $r_{1}(\omega) r_{2}(\omega)=\omega^{2}$ and $r_{1}(\omega)+r_{2}(\omega)=-(1+2(a+1 / a) \omega+$ $\left.\omega^{2}\right)$ as required.

The next lemma expresses the exponential decay of correlation in a pure gas phase. 
LEMMA 5.2. There are constants $C, c_{1}>0$ so that

$$
\left|\mathcal{G}_{\varepsilon, \varepsilon^{\prime}}(t)\right| \leq C e^{-c_{1}|t|}
$$

for all $t \in \mathbb{Z}$ and $\varepsilon, \varepsilon^{\prime} \in\{0,1\}$.

PROOF. We see from the proof of the previous lemma that $f_{\varepsilon, \varepsilon^{\prime}}(\omega)$ is an analytic function in the neighborhood of the unit circle. Let $t>0$ and take $r>1$, but close to 1 so that $f_{\varepsilon, \mathcal{E}^{\prime}}(\omega)$ is analytic in $\{\omega: 1 \leq|\omega| \leq r\}$. We see from (5.4) and Cauchy's theorem that

$$
\left|\mathcal{G}_{\varepsilon, \varepsilon^{\prime}}(t)\right|=\frac{a}{2 \pi}\left|\int_{\Gamma_{r}} \frac{f_{\varepsilon, \varepsilon^{\prime}}(\omega)}{\omega^{t}} \frac{d \omega}{\omega}\right| \leq \frac{C}{r^{t}} .
$$

If $t<0$, we take $r<1$ instead.

We are now ready to prove Lemma 4.1.

Proof OF LemMA 4.1. Let $(\bar{\delta}, \bar{k}, \bar{p}, \bar{q}) \in D_{r, 0}$ so that $\delta_{i}=0, p_{i}=p, q_{i}=q$, $k_{i}=k, 1 \leq i \leq r$. Thus,

$$
\begin{aligned}
T_{0}(m, r, \bar{\ell})= & \sum_{\bar{\varepsilon} \in\{0,1\}^{r}} \prod_{i=1}^{r}(-1)^{\ell_{i} \varepsilon_{i}} \sum_{k=1}^{M} \sum_{p=1}^{L_{2}} \sum_{q=1}^{L_{1}} w_{p, q}^{\ell_{1}+\cdots+\ell_{r}} \\
& \times \sum_{\bar{z} \in\left(\mathcal{L}_{m}\right)^{r}} \prod_{i=1}^{r} \mathbb{I}_{p, q, k}^{\varepsilon_{i}}\left(z_{i}\right) a \mathrm{i} \mathbb{K}_{1,1}^{-1}\left(x\left(z_{i+1}\right), y\left(z_{i}\right)\right)
\end{aligned}
$$

by (4.5) and (5.1). Recalling the definition of $A_{p, m}$ in (4.8) and using (5.3), we have that

$$
\begin{array}{r}
\sum_{\bar{z} \in\left(\mathcal{L}_{m}\right)^{r}} \prod_{i=1}^{r} \mathbb{I}_{p, q, k}^{\varepsilon_{i}}\left(z_{i}\right) a \mathrm{i} \mathbb{K}_{1,1}^{-1}\left(x\left(z_{i+1}\right), y\left(z_{i}\right)\right) \\
=\sum_{\bar{t} \in \mathbb{Z}^{r}} \prod_{i=1}^{r} \mathbb{I}_{A_{p, m}}\left(t_{i}\right) \mathcal{G}_{\varepsilon_{i}, \varepsilon_{i+1}}\left(t_{i+1}-t_{i}\right) .
\end{array}
$$

With the above equations and (4.6), we obtain

$$
\begin{aligned}
U_{0}(m)= & M \sum_{p=1}^{L_{2}} \sum_{q=1}^{L_{1}}\left(\sum_{s=1}^{\infty} \frac{w_{p, q}^{s}}{M^{s}} \sum_{r=1}^{s} \frac{(-1)^{r+1}}{r} \sum_{\substack{\ell_{1}+\cdots+\ell_{r}=s \\
\ell_{1}, \ldots, \ell_{r} \geq 1}} \frac{1}{\ell_{1} ! \cdots \ell_{r} !}\right. \\
& \left.\times \sum_{\bar{\varepsilon} \in\{0,1\}^{r}} \prod_{i=1}^{r}(-1)^{\ell_{i} \varepsilon_{i}} \sum_{\bar{t} \in \mathbb{Z}^{r}} \prod_{i=1}^{r} \mathbb{I}_{A_{p}, m}\left(t_{i}\right) \mathcal{G}_{\varepsilon_{i}, \varepsilon_{i+1}}\left(t_{i+1}-t_{i}\right)\right) .
\end{aligned}
$$


The result of the lemma now follows from (5.7) and the next claim, since we get the estimate

$$
\left|U_{0}(m)\right| \leq \frac{C}{M}
$$

ClaIM 1. There is a constant $C$ and an $R>0$ so that

$$
\begin{aligned}
& \mid \sum_{s=1}^{\infty} \frac{w^{s}}{M^{s}} \sum_{r=1}^{s} \frac{(-1)^{r+1}}{r} \sum_{\substack{\ell_{1}+\cdots+\ell_{r}=s \\
\ell_{1}, \ldots, \ell_{r} \geq 1}} \frac{1}{\ell_{1} ! \cdots \ell_{r} !} \\
& \times \sum_{\bar{\varepsilon} \in\{0,1\}^{r}} \sum_{\bar{t} \in \mathbb{Z}^{r}} \prod_{i=1}^{r}(-1)^{\ell_{i} \varepsilon_{i}} \mathbb{I}_{A_{p}, m}\left(t_{i}\right) \mathcal{G}_{\varepsilon_{i}, \varepsilon_{i+1}}\left(t_{i+1}-t_{i}\right) \\
& \leq \frac{C}{M^{2}}
\end{aligned}
$$

for $|w| \leq R$ and $1 \leq p \leq L_{2}$.

Proof of Claim 1. From (5.4), we see that

$$
\mathcal{G}_{\varepsilon, \varepsilon^{\prime}}(t)=a \mathrm{i}^{h\left(\varepsilon, \varepsilon^{\prime}\right)} \widehat{f}_{\varepsilon, \varepsilon^{\prime}}(t)
$$

for $t \in \mathbb{Z}$ where $\widehat{f}_{\varepsilon, \varepsilon^{\prime}}(t)$ is the $t$ th Fourier coefficient of $f_{\varepsilon, \varepsilon^{\prime}}$. Thus, we have

$$
\begin{aligned}
& \sum_{\bar{t} \in \mathbb{Z}^{r}} \prod_{i=1}^{r} \mathbb{I}_{A_{p}, m}\left(t_{i}\right) \mathcal{G}_{\varepsilon_{i}, \varepsilon_{i+1}}\left(t_{i+1}-t_{i}\right) \\
& \quad=\prod_{i=1}^{r} a \mathrm{i}^{h\left(\varepsilon_{i}, \varepsilon_{i+1}\right)} \sum_{\bar{t} \in \mathbb{Z}^{r}} \prod_{i=1}^{r} \mathbb{I}_{A_{p}, m}\left(t_{i}\right) \widehat{f}_{\varepsilon_{i}, \varepsilon_{i+1}}\left(t_{i+1}-t_{i}\right) .
\end{aligned}
$$

Using properties of convolutions of Fourier coefficients, we have

$$
\begin{aligned}
\sum_{t_{2}, \ldots, t_{r} \in \mathbb{Z}} \prod_{i=1}^{r}{\widehat{f_{\varepsilon_{i}}, \varepsilon_{i+1}}}\left(t_{i+1}-t_{i}\right) \\
=\sum_{t_{r} \in \mathbb{Z}}\left(\hat{f}_{\varepsilon_{1}, \varepsilon_{2}} \widehat{\cdots f_{\varepsilon_{r-1}, \varepsilon_{r}}}\right)\left(t_{r}-t_{1}\right) \widehat{f}_{\varepsilon_{r}, \varepsilon_{1}}\left(t_{1}-t_{r}\right) \\
=\sum_{t_{r} \in \mathbb{Z}}\left(f_{\varepsilon_{1}, \varepsilon_{2}} \widehat{\cdots f_{\varepsilon_{r-1}}, \varepsilon_{r}}\right)\left(t_{r}\right) \widehat{f}_{\varepsilon_{r}, \varepsilon_{1}}\left(-t_{r}\right) \\
=\left(f_{\varepsilon_{1}, \varepsilon_{2}} \widehat{\cdots f_{\varepsilon_{r-1}, \varepsilon_{r}}}\right)(0) \\
=\frac{1}{2 \pi \mathrm{i}} \int_{\Gamma_{1}} \frac{d \omega}{\omega} \prod_{i=1}^{r} f_{\varepsilon_{i}, \varepsilon_{i+1}}(\omega)
\end{aligned}
$$


for $r \geq 2$. Thus, for $r \geq 2$ we have

$$
\begin{aligned}
\mid \sum_{\bar{t} \in \mathbb{Z}^{r}} & \prod_{i=1}^{r} \mathbb{I}_{A_{p, m}}\left(t_{i}\right) \widehat{f}_{\varepsilon_{i}, \varepsilon_{i+1}}\left(t_{i+1}-t_{i}\right)-\frac{\left|A_{p, m}\right|}{2 \pi \mathrm{i}} \int_{\Gamma_{1}} \frac{d \omega}{\omega} \prod_{i=1}^{r} f_{\varepsilon_{i}, \varepsilon_{i+1}}(\omega) \mid \\
= & \left|\sum_{\bar{t} \in \mathbb{Z}^{r}} \mathbb{I}_{A_{p, m}}\left(t_{1}\right)\left(\prod_{i=2}^{r} \mathbb{I}_{A_{p, m}}\left(t_{i}\right)-1\right) \prod_{i=1}^{r} \widehat{f}_{\varepsilon_{i}, \varepsilon_{i+1}}\left(t_{i+1}-t_{i}\right)\right| \\
& \leq \sum_{\bar{t} \in \mathbb{Z}^{r}} \mathbb{I}_{A_{p, m}}\left(t_{1}\right)\left(\sum_{j=2}^{r} \mathbb{I}_{A_{p, m}^{c}}\left(t_{j}\right)\right) \prod_{i=1}^{r} C e^{-c_{1}\left|t_{i+1}-t_{i}\right|}
\end{aligned}
$$

by Lemma 5.2 and equation (5.9).

Introduce new coordinates $s_{1}=t_{1}, s_{i}=t_{i}-t_{i-1}, 2 \leq i \leq r$. The inverse is

$$
t_{j}=\sum_{i=1}^{j} s_{i}
$$

so we get a bijection from $\mathbb{Z}^{r}$ to $\mathbb{Z}^{r}$. We see that the right-hand side in (5.10) is less than or equal to

$$
\begin{aligned}
& C^{r} \sum_{\bar{s} \in \mathbb{Z}^{r}} \mathbb{I}_{A_{p, m}}\left(s_{1}\right)\left(\sum_{j=2}^{r} \mathbb{I}_{A_{p, m}^{c}}\left(s_{1}+\cdots+s_{j}\right)\right) \prod_{i=1}^{r} C e^{-c_{1} \sum_{i=2}^{r}\left|s_{i}\right|-c_{1}\left|s_{2}+\cdots+s_{r}\right|} \\
& \leq C^{r} \sum_{j=2}^{r} \sum_{\sigma, s_{1} \in \mathbb{Z}} \sum_{\substack{s_{2}, \ldots, s_{r} \in \mathbb{Z} \\
s_{2}+\cdots+s_{j}=\sigma}} \mathbb{I}_{A_{p, m}}\left(s_{1}\right) \mathbb{I}_{A_{p, m}^{c}}\left(s_{1}+\sigma\right) e^{-\frac{c_{1}}{2}|\sigma|-\frac{c_{1}}{2} \sum_{i=2}^{r}\left|s_{i}\right|} \\
& \leq C^{r} \sum_{j=2}^{r} \sum_{\sigma, s_{1} \in \mathbb{Z}} \mathbb{I}_{A_{p, m}}\left(s_{1}\right) \mathbb{I}_{A_{p, m}^{c}}\left(s_{1}+\sigma\right) e^{-\frac{c_{1}}{2}|\sigma|}\left(\sum_{s_{2}, \ldots, s_{r} \in \mathbb{Z}} e^{-\frac{c_{1}}{2}\left(\left|s_{2}\right|+\cdots+\left|s_{r}\right|\right)}\right) \\
& \leq C^{r} \sum_{\sigma, s_{1} \in \mathbb{Z}} \mathbb{I}_{A_{p, m}}\left(s_{1}\right) \mathbb{I}_{A_{p, m}^{c}}\left(s_{1}+\sigma\right) e^{-\frac{c_{1}}{2}|\sigma|} \leq C^{r} .
\end{aligned}
$$

Thus, we find

$$
\left|\sum_{\bar{t} \in \mathbb{Z}^{r}} \prod_{i=1}^{r} \mathbb{I}_{A_{p, m}}\left(t_{i}\right) \widehat{f}_{\varepsilon_{i}, \varepsilon_{i+1}}\left(t_{i+1}-t_{i}\right)-\frac{\left|A_{p, m}\right|}{2 \pi \mathrm{i}} \int_{\Gamma_{1}} \frac{d \omega}{\omega} \prod_{i=1}^{r} f_{\varepsilon_{i}, \varepsilon_{i+1}}(\omega)\right| \leq C^{r}
$$

Write

$$
\begin{aligned}
\Sigma_{1} & =\sum_{s=1}^{\infty} \frac{w^{s}}{M^{s}} \frac{1}{s !} \sum_{\varepsilon_{1} \in\{0,1\}} \sum_{t_{1} \in \mathbb{Z}}(-1)^{s \varepsilon_{1}} \mathbb{I}_{A_{p, m}}\left(t_{1}\right) a \mathrm{i}^{h\left(\varepsilon_{1}, \varepsilon_{1}\right)} \widehat{f}_{\varepsilon_{1}, \varepsilon_{1}}(0) \\
& =\sum_{s=1}^{\infty} \frac{w^{s}}{M^{s}} \frac{1}{s !} \sum_{\varepsilon_{1} \in\{0,1\}}(-1)^{s \varepsilon_{1}} \frac{\left|A_{p, m}\right|}{2 \pi \mathrm{i}} \int_{\Gamma_{1}} \frac{d \omega}{\omega} a \mathrm{i}^{h\left(\varepsilon_{1}, \varepsilon_{1}\right)} f_{\varepsilon_{1}, \varepsilon_{1}}(\omega)
\end{aligned}
$$


and

$$
\begin{aligned}
\Sigma_{2}= & \sum_{s=2}^{\infty} \frac{w^{s}}{M^{s}} \sum_{r=2}^{s} \frac{(-1)^{r+1}}{r} \sum_{\substack{\ell_{1}+\cdots+\ell_{r}=s \\
\ell_{1}, \ldots, \ell_{r} \geq 1}} \frac{1}{\ell_{1} ! \cdots \ell_{r} !} \sum_{\bar{\varepsilon} \in\{0,1\}^{r}} \sum_{\bar{t} \in \mathbb{Z}^{r}} \\
& \times \prod_{i=1}^{r}(-1)^{\ell_{i} \varepsilon_{i}} \mathbb{I}_{A_{p, m}}\left(t_{i}\right) a \mathrm{i}^{h\left(\varepsilon_{i}, \varepsilon_{i+1}\right)} \hat{f}_{\varepsilon_{i}, \varepsilon_{i+1}}\left(t_{i+1}-t_{i}\right),
\end{aligned}
$$

so that the left-hand side of (5.8) is $\left|\Sigma_{1}+\Sigma_{2}\right|$. Now, using (5.11), we see that

$$
\begin{aligned}
\mid \Sigma_{2} & -\sum_{s=2}^{\infty} \frac{w^{s}}{M^{s}} \sum_{r=2}^{s} \frac{(-1)^{r+1}}{r} \sum_{\substack{\ell_{1}+\cdots+\ell_{r}=s \\
\ell_{1}, \ldots, \ell_{r} \geq 1}} \frac{1}{\ell_{1} ! \cdots \ell_{r} !} \sum_{\bar{\varepsilon} \in\{0,1\}^{r}} \frac{\left|A_{p, m}\right|}{2 \pi \mathrm{i}} \int_{\Gamma_{1}} \frac{d \omega}{\omega} \\
\times & \prod_{i=1}^{r}(-1)^{\ell_{i} \varepsilon_{i}} a \mathrm{i}^{h\left(\varepsilon_{i}, \varepsilon_{i+1}\right)} f_{\varepsilon_{i}, \varepsilon_{i+1}}(\omega) \mid \\
& \leq \sum_{s=2}^{\infty} \frac{|w|^{s}}{M^{s}} \sum_{r=2}^{s} \frac{1}{r} \sum_{\substack{\ell_{1}+\cdots+\ell_{r}=s \\
\ell_{1}, \ldots, \ell_{r} \geq 1}} \frac{C^{r}}{\ell_{1} ! \cdots \ell_{r} !} \leq \sum_{s=2}^{\infty} \frac{R^{s} C^{s}}{M^{s}} \leq \frac{C}{M^{2}}
\end{aligned}
$$

if $R$ is sufficiently small. Thus,

$$
\begin{aligned}
\mid \Sigma_{1}+ & \Sigma_{2} \mid \\
\leq & \mid \sum_{s=1}^{\infty} \frac{w^{s}}{M^{s}} \sum_{r=1}^{s} \frac{(-1)^{r+1}}{r} \sum_{\substack{\ell_{1}+\cdots+\ell_{r}=s \\
\ell_{1}, \ldots, \ell_{r} \geq 1}} \frac{\left|A_{p, m}\right|}{\ell_{1} ! \cdots \ell_{r} !} \sum_{\bar{\varepsilon} \in\{0,1\}^{r}} \frac{1}{2 \pi \mathrm{i}} \int_{\Gamma_{1}} \frac{d \omega}{\omega} \\
& \times \prod_{i=1}^{r}(-1)^{\ell_{i} \varepsilon_{i}} a \mathrm{i}^{h\left(\varepsilon_{i}, \varepsilon_{i+1}\right)} f_{\varepsilon_{i}, \varepsilon_{i+1}}(\omega) \mid+\frac{C}{M^{2}} .
\end{aligned}
$$

Let $F_{\omega}=\left(F_{\omega}\left(\varepsilon_{1}, \varepsilon_{2}\right)\right)_{0 \leq \varepsilon_{1}, \varepsilon_{2} \leq 1}$ be the two-by-two matrix with elements $F_{\omega}\left(\varepsilon_{1}\right.$, $\left.\varepsilon_{2}\right)=a \mathrm{i}^{h\left(\varepsilon_{1}, \varepsilon_{2}\right)} f_{\varepsilon_{1}, \varepsilon_{2}}(\omega)$ for $0 \leq \varepsilon_{1}, \varepsilon_{2} \leq 1$, and let $\eta\left(\varepsilon_{1}\right)=(-1)^{\varepsilon_{1}}$. Then the expression between the absolute value signs in the right-hand side of (5.12) can be written as

$$
\begin{aligned}
& \frac{\left|A_{p, m}\right|}{2 \pi \mathrm{i}} \int_{\Gamma_{1}} \frac{d \omega}{\omega} \sum_{s=1}^{\infty} \frac{w^{s}}{M^{s}} \sum_{r=1}^{s} \frac{(-1)^{r+1}}{r} \\
& \quad \times \sum_{\substack{\ell_{1}+\cdots+\ell_{r}=s \\
\ell_{1}, \ldots, \ell_{r} \geq 1}} \frac{1}{\ell_{1} ! \cdots \ell_{r} !} \operatorname{tr}\left(\eta^{\ell_{1}} F_{\omega} \cdots \eta^{\ell_{r}} F_{\omega}\right) .
\end{aligned}
$$

Here, we view $F_{\omega}$ as an operator with kernel $F_{\omega}$ and on functions $\{0,1\} \rightarrow \mathbb{C}$, that is, the trace is for a product of two $2 \times 2$ matrices. The expression in the integrand 
above is a cumulant expansion of $\log \operatorname{det}\left(\mathbb{I}+\left(e^{\frac{\omega}{M} \eta}-1\right) F_{\omega}\right)$. This means that $(5.13)$ equals

$$
\frac{\left|A_{p, m}\right|}{2 \pi \mathrm{i}} \int_{\Gamma_{1}} \frac{d \omega}{\omega} \log \operatorname{det}\left(\mathbb{I}+\left(e^{\frac{\omega}{M} \eta}-1\right) F_{\omega}\right)
$$

provided that $R$ is small enough. The above determinant can be written explicitly and is given by

$$
\begin{aligned}
\operatorname{det}( & \left.\left(\begin{array}{ll}
1 & 0 \\
0 & 1
\end{array}\right)+\left(\begin{array}{cc}
\left(e^{\frac{w}{M}}-1\right) a f_{0,0}(\omega) & \left(e^{\frac{w}{M}}-1\right) a \mathrm{i} f_{0,1}(\omega) \\
\left(e^{-\frac{w}{M}}-1\right) a \mathrm{i} f_{1,0}(\omega) & \left(e^{-\frac{w}{M}}-1\right) a f_{1,1}(\omega)
\end{array}\right)\right) \\
= & \left(1+\left(e^{\frac{w}{M}}-1\right) a f_{0,0}(\omega)\right)\left(1+\left(e^{-\frac{w}{M}}-1\right) a f_{1,1}(\omega)\right) \\
& +\left(e^{\frac{w}{M}}-1\right)\left(e^{-\frac{w}{M}}-1\right) a^{2} f_{0,1}(\omega) f_{1,0}(\omega) \\
= & 1+\left(e^{\frac{w}{M}}-1\right) a f_{0,0}(\omega)+\left(e^{-\frac{w}{M}}-1\right) a f_{1,1}(\omega) \\
& +a^{2}\left(2-e^{\frac{w}{M}}-e^{-\frac{w}{M}}\right) f_{0,0}(\omega) f_{1,1}(\omega) \\
& +a^{2}\left(2-e^{\frac{w}{M}}-e^{-\frac{w}{M}}\right) f_{0,1}(\omega) f_{1,0}(\omega) \\
= & +\left(e^{\frac{w}{M}}-1\right) a f_{0,0}(\omega)+\left(e^{-\frac{w}{M}}-1\right) a f_{0,0}(\omega) \\
& +a^{2}\left(2-e^{\frac{w}{M}}-e^{-\frac{w}{M}}\right) f_{0,0}(\omega)^{2} \\
& +a^{2}\left(2-e^{\frac{w}{M}}-e^{-\frac{w}{M}}\right) f_{0,1}(\omega) f_{1,0}(\omega) \\
= & +\left(e^{\frac{w}{M}}-1\right) a f_{0,0}(\omega)+\left(e^{-\frac{w}{M}}-1\right) a f_{0,0}(\omega) \\
& +a\left(2-e^{\frac{w}{M}}-e^{-\frac{w}{M}}\right) f_{0,0}(\omega)
\end{aligned}
$$

where the third equality follows from the first relation in Lemma 5.1 and the fourth equality follows from the second relation in Lemma 5.1. We conclude that

$$
\operatorname{det}\left(\mathbb{I}+\left(e^{\frac{w}{M} \eta}-1\right) F_{\omega}\right)=1
$$

and so we have shown that $\left|\Sigma_{1}+\Sigma_{2}\right| \leq C / M^{2}$. This proves the claim.

The proof of the claim concludes the proof of Lemma 4.1 .

We now give the proof of Lemma 4.6.

ProOf OF LEMma 4.6. We have that from (4.7) and (4.12)

$$
Q(\bar{\varepsilon})=P(\bar{\varepsilon}, \overline{1})=\prod_{i=1}^{s} \frac{a \mathrm{i}}{2} \sqrt{1-2 c} \mathrm{~g}_{\varepsilon_{1}, \varepsilon_{i+1}} \mathcal{C}^{\varepsilon_{i}+\varepsilon_{i+1}-2}
$$


From this, we see that the left-hand side of (4.6) is the trace of the $s$ th power of a two-by-two matrix where the $\left(\varepsilon_{1}+1, \varepsilon_{2}+1\right)$ th entry is

$$
a \mathrm{i} \frac{\sqrt{1-2 c}}{2} \frac{g_{\varepsilon_{1}, \varepsilon_{2}}}{\mathcal{C}^{2-\varepsilon_{1}-\varepsilon_{2}}}
$$

for $\varepsilon_{1}, \varepsilon_{2} \in\{0,1\}$. These entries are simplified using the expressions of $g_{\varepsilon_{1}, \varepsilon_{2}}$ and $\mathcal{C}$ given above. Thus, the two-by-two matrix has the explicit form

$$
\left(\begin{array}{cc}
-\frac{1}{2}\left(\begin{array}{cc}
1+\frac{1}{\sqrt{a^{2}+1}}
\end{array}\right) & \frac{a \mathrm{i}}{2 \sqrt{a^{2}+1}} \\
-\frac{a \mathrm{i}}{2 \sqrt{a^{2}+1}} & -\frac{1}{2}+\frac{1}{2 \sqrt{a^{2}+1}}
\end{array}\right)
$$

which has eigenvalues 0 and -1 , as required.

6. Proof of Proposition 3.1. In this section, we give the proof of Proposition 3.1. In order to give this proof, we rely on various results from [9] which are recalled below.

Let $\alpha_{x}, \alpha_{y}, \beta_{x}, \beta_{y} \in \mathbb{R}, k_{x}, k_{y} \in \mathbb{Z}$ and $f_{x}, f_{y} \in \mathbb{Z}^{2}$. Set

$$
\begin{aligned}
x= & \left(\rho_{m}+2\left[\alpha_{x} \lambda_{1}(2 m)^{1 / 3}\right]\right) e_{1} \\
& -\left(2\left[\beta_{x} \lambda_{2}(2 m)^{2 / 3}+k_{x} \lambda_{2}(\log m)^{2}\right]\right) e_{2}+f_{x}, \\
y= & \left(\rho_{m}+2\left[\alpha_{y} \lambda_{1}(2 m)^{1 / 3}\right]\right) e_{1} \\
& -\left(2\left[\beta_{y} \lambda_{2}(2 m)^{2 / 3}+k_{y} \lambda_{2}(\log m)^{2}\right]\right) e_{2}+f_{y} .
\end{aligned}
$$

From [9], Theorem 2.7, and its proof, we have the following.

THEOREM 6.1 ([9]). Assume that $x \in \mathrm{W}_{\varepsilon_{x}}$ and $y \in \mathrm{B}_{\varepsilon_{y}}$ are given by (6.1) with $\varepsilon_{x}, \varepsilon_{y} \in\{0,1\}$. Furthermore, assume that $\left|\alpha_{x}\right|,\left|\alpha_{y}\right|,\left|\beta_{x}\right|,\left|\beta_{y}\right|,\left|f_{x}\right|,\left|f_{y}\right| \leq C$ for some constant $C>0$ and that $\left|k_{x}\right|,\left|k_{y}\right| \leq M$. Then, as $m \rightarrow \infty$

$$
\begin{aligned}
\mathbb{K}_{\mathrm{A}}(x, y)= & \mathrm{i}^{y_{1}-x_{1}+1} \mathcal{C}^{\frac{-2-x_{1}+x_{2}+y_{1}-y_{2}}{2}} c_{0 g_{\varepsilon_{x}}, \varepsilon_{y}} e^{\alpha_{y} \beta_{y}-\alpha_{x} \beta_{x}-\frac{2}{3}\left(\beta_{x}^{3}-\beta_{y}^{3}\right)} \\
& \times(2 m)^{-\frac{1}{3}}\left(\tilde{\mathcal{A}}\left(\beta_{x}, \alpha_{x}+\beta_{x}^{2} ; \beta_{y}, \alpha_{y}+\beta_{y}^{2}\right)+o(1)\right) .
\end{aligned}
$$

Also, as $m \rightarrow \infty$,

$$
\begin{aligned}
\mathbb{K}_{1,1}^{-1}(x, y)= & \mathrm{i}^{y_{1}-x_{1}+1} \mathcal{C}^{\frac{-2-x_{1}+x_{2}+y_{1}-y_{2}}{2}} \operatorname{cog}_{\varepsilon_{x}, \varepsilon_{y}} e^{\alpha_{y} \beta_{y}-\alpha_{x} \beta_{x}-\frac{2}{3}\left(\beta_{x}^{3}-\beta_{y}^{3}\right)} \\
& \times(2 m)^{-\frac{1}{3}}\left(\phi_{\beta_{x}, \beta_{y}}\left(\alpha_{x}+\beta_{x}^{2} ; \alpha_{y}+\beta_{y}^{2}\right)+o(1)\right) .
\end{aligned}
$$

REMARK 2. The difference between the above version of the theorem and the statement given in [9], Theorem 2.7, is that there is a positional change of the vertices $x$ and $y$ by at most $\left|k_{x} \lambda_{2}(\log m)^{2}\right|$ and $\left|k_{y} \lambda_{2}(\log m)^{2}\right|$ and the reverse 
time orientation, which simply consists of the change $\beta_{x} \mapsto-\beta_{x}$ and $\beta_{y} \mapsto-\beta_{y}$. By comparing the statement of [9], Theorem 2.7, and Theorem 6.1, the positional change affects the exponent of $\mathcal{C}$ and the error term, where we remind the reader that $|G(\mathrm{i})|$ in [9] is equal to $\mathcal{C}$ in this paper.

More explicitly, this positional change only alters the Taylor series computation of the ratio $H_{x_{1}+1, x_{2}}\left(\omega_{1}\right) / H_{y_{1}, y_{2}+1}\left(\omega_{2}\right)$ using the local change of variables [9], equation (3.22), where $H_{x_{1}, x_{2}}(\omega)$ is defined in [9] and $x=\left(x_{1}, x_{2}\right)$ and $y=\left(y_{1}, y_{2}\right)$ are as defined in (6.1). Catering for this alteration immediately gives Theorem 6.1.

As given in [9], (4.20), define

$$
E_{k, l}=\frac{1}{(2 \pi \mathrm{i})^{2}} \int_{\Gamma_{1}} \frac{d u_{1}}{u_{1}} \int_{\Gamma_{1}} \frac{d u_{2}}{u_{2}} \frac{1}{\tilde{c}\left(u_{1}, u_{2}\right) u_{1}^{k} u_{2}^{l}} .
$$

Then (see [9], equation (4.22)), for $x \in \mathrm{W}_{\varepsilon_{x}}, y \in \mathrm{B}_{\varepsilon_{y}}$,

$$
\mathbb{K}_{1,1}^{-1}(x, y)=-\mathrm{i}^{1+h\left(\varepsilon_{x}, \varepsilon_{y}\right)}\left(a^{\varepsilon_{y}} E_{\mathrm{k}_{1}, 1_{1}}+a^{1-\varepsilon_{y}} E_{\mathrm{k}_{2}, 1_{2}}\right),
$$

where

$$
\begin{aligned}
& \mathrm{k}_{1}=\frac{x_{2}-y_{2}-1}{2}+h\left(\varepsilon_{x}, \varepsilon_{y}\right), \quad \mathrm{k}_{2}=\frac{x_{2}-y_{2}+1}{2}-h\left(\varepsilon_{x}, \varepsilon_{y}\right), \\
& \mathrm{l}_{1}=\frac{y_{1}-x_{1}-1}{2}, \quad \mathrm{I}_{2}=\frac{y_{1}-x_{1}+1}{2} .
\end{aligned}
$$

From [9], Lemma 4.6 and Lemma 4.7, we get the following asymptotic formulas and estimates.

Lemma 6.2 ([9]). Let $A_{m}, B_{m}, m \geq 1$ be given and set $b_{m}=\max \left(\left|A_{m}\right|,\left|B_{m}\right|\right)$, and let $a_{m}=A_{m}$ if $b_{m}=\left|B_{m}\right|$, and let $a_{m}=B_{m}$ if $b_{m}=\left|A_{m}\right|$ :

1. Assume that $b_{m} \rightarrow \infty$ as $m \rightarrow \infty$ and $\left|a_{m}\right| \leq b_{m}^{7 / 12}$ for large $m$. Then there exists a constant $d_{1}>0$ so that

$$
E_{B_{m}+A_{m}, B_{m}-A_{m}}
$$

$$
=\frac{(-1)^{a_{m}+b_{m}} \mathcal{C}^{2 b_{m}}\left(e^{-\frac{\sqrt{1-2 c}}{2 c} \frac{a_{m}^{2}}{b_{m}}}\left(1+O\left(b_{m}^{-1 / 4}\right)\right)+O\left(e^{-d_{1} b_{m}^{1 / 6}}\right)\right)}{2\left(1+a^{2}\right)(1-2 c)^{1 / 4} \sqrt{2 \pi c b_{m}}}
$$

as $m \rightarrow \infty$.

2. Assume that $b_{m}>0, m \geq 1$. There exist constants $C, d_{1}, d_{2}>0$ so that

$$
\left|E_{B_{m}+A_{m}, B_{m}-A_{m}}\right| \leq \frac{C}{\sqrt{b_{m}}} \mathcal{C}^{2 b_{m}}\left(e^{-d_{1} \frac{a_{m}^{2}}{b_{m}}}+e^{-d_{2} b_{m}}\right)
$$

for all $m \geq 1$. 
Motivated by (6.4) and (6.6), we define

$$
A_{m, i}=\frac{\mathrm{k}_{i}-\mathrm{I}_{i}}{2} \quad \text { and } \quad B_{m, i}=\frac{\mathrm{k}_{i}+\mathrm{l}_{i}}{2}
$$

for $i \in\{1,2\}$. It follows from (6.5) that

$$
\begin{aligned}
& 2 A_{m, i}=\frac{x_{1}\left(z^{\prime}\right)+x_{2}\left(z^{\prime}\right)-\left(y_{1}(z)+y_{2}(z)\right)}{2}-(-1)^{i} h\left(\varepsilon(z), \varepsilon\left(z^{\prime}\right)\right), \\
& 2 B_{m, i}=\frac{x_{2}\left(z^{\prime}\right)-x_{1}\left(z^{\prime}\right)+\left(y_{1}(z)-y_{2}(z)\right)}{2}+(-1)^{i}\left(1-h\left(\varepsilon(z), \varepsilon\left(z^{\prime}\right)\right)\right) .
\end{aligned}
$$

If we have $z \in \mathcal{L}_{m}(q, k), z^{\prime} \in \mathcal{L}_{m}\left(q^{\prime}, k^{\prime}\right), t=t(z), t^{\prime}=t\left(z^{\prime}\right), \varepsilon=\varepsilon(z)$ and $\varepsilon^{\prime}=$ $\varepsilon\left(z^{\prime}\right)$, then using (1.7) and (2.1)

$$
\begin{aligned}
& 2 A_{m, i}=2\left(t^{\prime}-\tau_{m}\left(q^{\prime}\right)\right)-2\left(t-\tau_{m}(q)\right)+2\left(\varepsilon-\varepsilon^{\prime}\right)-(-1)^{i} h\left(\varepsilon, \varepsilon^{\prime}\right), \\
& 2 B_{m, i}=\beta_{m}(q, k)-\beta_{m}\left(q^{\prime}, k^{\prime}\right)+\varepsilon+\varepsilon^{\prime}-1+(-1)^{i}\left(1-h\left(\varepsilon, \varepsilon^{\prime}\right)\right) .
\end{aligned}
$$

We are now ready for the proof of Proposition 3.1.

ProOf of Proposition 3.1. To prove part (1) in the statement of the proposition, we apply Theorem 6.1. By comparing (1.7) and (6.1), $y=y(z)$, we see that

$$
\alpha_{y}=\frac{t-\tau_{m}(q)}{\lambda_{1}(2 m)^{1 / 3}} \quad \text { and } \quad \beta_{y}=\beta_{q},
$$

if $z \in \mathcal{L}_{m}(q, k), t=t(z)$, where we have disregarded integer parts. Thus, we have

$$
\alpha_{y} \beta_{y}+\frac{2}{3} \beta_{y}^{3}=\frac{t}{\lambda_{1}(2 m)^{1 / 3}} \beta_{q}-\frac{1}{3} \beta_{q}^{3}=\gamma_{1}(z)
$$

by (3.4). Using (3.3), (3.5) and (6.10), we see that part (1) in the statement of the proposition follows from (6.2). Similarly, part (2) in the statement of the proposition follows from (6.3).

We now consider part (3) in the statement of the proposition, that is, $q=q^{\prime}$, $k>k^{\prime}$. From (6.9) and the definition of $\beta_{m}(q, k)$, we see that

$$
B_{m, i}=\left(k-k^{\prime}\right) \lambda_{2}(\log m)^{2}+\frac{1}{2}\left(\varepsilon+\varepsilon^{\prime}+(-1)^{i}\left(1-h\left(\varepsilon_{1}, \varepsilon_{2}\right)\right)\right)
$$

so $B_{m, i}>0$ if $m$ is sufficiently large. Also,

$$
A_{m, i}=t^{\prime}-t+\varepsilon-\varepsilon^{\prime},
$$

since $t_{m}\left(q^{\prime}\right)=t_{m}(q)$. Assume now that $\left|t^{\prime}-t\right| \leq c_{2}\left(\left(k-k^{\prime}\right)(\log m)^{2}\right)^{7 / 12}$. Then $b_{m, i}=\left|B_{m, i}\right|$ and

$$
\left|a_{m, i}\right|=\left|A_{m, i}\right| \leq b_{m, i}^{7 / 12}
$$


for large $m$ if $c_{2}<1$. By (6.6),

$$
\begin{aligned}
E_{\mathrm{k}_{i}, 1_{i}}= & \frac{(-1)^{k_{i}} \mathcal{C}^{b_{m, i}}}{2\left(1+a^{2}\right)(1-2 c)^{1 / 4} \sqrt{2 \pi c b_{m, i}}} \\
& \times\left(e^{-\frac{\sqrt{1-2 c}}{2 c} \frac{A_{m, i}^{2}}{B_{m, i}}}\left(1+O\left(b_{m, i}^{-1 / 4}\right)\right)+O\left(e^{-d_{1} b_{m, i}^{1 / 6}}\right)\right) .
\end{aligned}
$$

Note that

$$
-\frac{\sqrt{1-2 c}}{2 c} \frac{A_{m, i}^{2}}{B_{m, i}}=-\frac{\lambda_{1}^{2} \sqrt{1-2 c}}{2 c \lambda_{2}\left(k-k^{\prime}\right)}\left(\frac{t^{\prime}}{\lambda_{1} \log m}-\frac{t}{\lambda_{1} \log m}\right)^{2}+o(1)
$$

and that

$$
\left|\gamma_{1}\left(z^{\prime}\right)-\gamma_{1}(z)\right|=\left|\frac{t^{\prime}-t}{\lambda_{1}(2 m)^{1 / 3}} \beta_{q}\right| \leq C
$$

since $\left|t^{\prime}-t\right| \leq C m^{1 / 3}$. We can now use (3.3), (3.8), (6.4) and proceed as in the proof of [9], Proposition 3.4, and this will give part (3)(a) in the proposition.

We turn now to part (3)(b) in the proposition. Consider (3.8) and note that

$$
\mathcal{C}^{\gamma_{2}\left(z^{\prime}\right)-\gamma_{2}(z)+2-2 \varepsilon^{\prime}}=\mathcal{C}^{\frac{1}{2}\left(x_{1}\left(z^{\prime}\right)-x_{2}\left(z^{\prime}\right)+y_{2}(z)-y_{1}(z)+2\right)}=\mathcal{C}^{-2 B_{m, i}+1-(-1)^{i}\left(1-h\left(\varepsilon, \varepsilon^{\prime}\right)\right)}
$$

by (3.7) and (6.8). We can now use (6.7) to get

$$
\left|E_{\mathrm{k}_{i}, 1_{i}}\right| \leq \frac{C}{\sqrt{b_{m, i}}} \mathcal{C}^{2 b_{m, i}}\left(e^{-d_{1} \frac{a_{m, i}^{2}}{b_{m, i}}}+e^{-d_{2} b_{m, i}}\right) .
$$

If $\left(c_{2}\left(\left(k-k^{\prime}\right)\right)(\log m)^{2}\right)^{7 / 12} \leq\left|t^{\prime}-t\right| \leq \lambda_{2}\left(k-k^{\prime}\right)(\log m)^{2}$, then $b_{m, i}=B_{m, i}$. The estimate (6.11) holds and combining these facts, we obtain the bound in (3)(b) in the statement of the proposition.

If $\left|t^{\prime}-t\right| \geq \lambda_{2}\left(k-k^{\prime}\right)(\log m)^{2}$, then

$$
\begin{aligned}
& b_{m, i}=\left|A_{m, i}\right|=\left|t^{\prime}-t\right|+O(1), \\
& a_{m, i}=B_{m, i}=\left(k-k^{\prime}\right) \lambda_{2}(\log m)^{2}+O(1) .
\end{aligned}
$$

It follows, since $\left|b_{m, 2}-b_{m, 1}\right|$ and $\left|a_{m, 2}-a_{m, 1}\right|$ are bounded, that

$$
\left|\mathcal{K}_{m, 0}\left(z, z^{\prime}\right)\right| \leq C \mathcal{C}^{2\left(b_{m, 1}-B_{m, 1}\right)} e^{c_{1} \frac{-a_{m, 1}^{2}}{b_{m, 1}}} .
$$

If $\lambda_{2}\left(k-k^{\prime}\right)(\log m)^{2} \leq\left|t^{\prime}-t\right| \leq 2 \lambda_{2}\left(k-k^{\prime}\right)(\log m)^{2}$, we can use $\mathcal{C}<1$ and $b_{m, 1}-$ $B_{m, 1} \geq 0$, to get

$$
\left|\mathcal{K}_{m, 0}\left(z, z^{\prime}\right)\right| \leq e^{-c_{1}\left(k-k^{\prime}\right)(\log m)^{2}} .
$$

If $\left|t^{\prime}-t\right|>2 \lambda_{2}\left(k-k^{\prime}\right)(\log m)^{2}$, we use $\mathcal{C}<1$ to get

$$
\left|\mathcal{K}_{m, 0}\left(z, z^{\prime}\right)\right| \leq C \mathcal{C}^{2\left(b_{m, 1}-B_{m, 1}\right)} \leq C \mathcal{C}^{2\left(k-k^{\prime}\right) \lambda_{2}(\log m)^{2}} \leq C e^{-c_{1}\left(k-k^{\prime}\right)(\log m)^{2}}
$$


with an appropriate $c_{1}>0$. In either case, we have shown (3)(c) in the statement of the proposition.

Consider now the case (4) in the statement of the proposition. In this case, $B_{m, i}<0$ and we see that the factor

$$
\mathcal{C}^{2\left(b_{m, i}-B_{m, i}\right)}
$$

in (6.12) will give us the decay we need in order to prove the bound in statement (4) of the proposition.

Finally, we consider statement (5) in the proposition, that is, $q=q^{\prime}$ and $k=k^{\prime}$. Then we have

$$
B_{m, i}=\varepsilon+\varepsilon^{\prime}-1+(-1)^{i}\left(1-h\left(\varepsilon, \varepsilon^{\prime}\right)\right)
$$

and

$$
A_{m, i}=t^{\prime}-t+\varepsilon-\varepsilon^{\prime} .
$$

Thus, if $\left|t^{\prime}-t\right|$ is sufficiently large, then $b_{m, i}=\left|A_{m, i}\right|$ and $a_{m, i}=B_{m, i}$. Since $\left|B_{m, i}\right| \leq 2$,

$$
2\left(b_{m, i}-B_{m, i}\right) \geq 2\left(\left|t^{\prime}-t\right|-2\right)
$$

and again the factor in (6.13) gives the desired bound.

Acknowledgements. All the authors wish to thank the Galileo Galilei Institute for hospitality and support during the scientific program "Statistical Mechanics, Integrability and Combinatorics", which provided a useful platform for this work. We would also like to thank Anton Bovier, Maurice Duits and Patrik Ferrari for useful discussions and the referees for useful comments and suggestions.

\section{REFERENCES}

[1] Borodin, A. and Ferrari, P. L. (2014). Anisotropic growth of random surfaces in $2+1$ dimensions. Comm. Math. Phys. 325 603-684. MR3148098

[2] Borodin, A. and Rains, E. M. (2005). Eynard-Mehta theorem, Schur process, and their Pfaffian analogs. J. Stat. Phys. 121 291-317. MR2185331

[3] Boutillier, C. (2007). Pattern densities in non-frozen planar dimer models. Comm. Math. Phys. 271 55-91.

[4] Boutillier, C., Bouttier, J., Chapuy, G., Corteel, S. and Ramassamy, S. (2017). Dimers on rail yard graphs. Ann. Inst. Henri Poincaré D 4 479-539. MR3734415

[5] Bouttier, J., Chapuy, G. and Corteel, S. (2017). From Aztec diamonds to pyramids: Steep tilings. Trans. Amer. Math. Soc. 369 5921-5959. MR3646784

[6] Breuer, J. and Duits, M. (2014). The Nevai condition and a local law of large numbers for orthogonal polynomial ensembles. Adv. Math. 265 441-484. MR3255467

[7] Bufetov, A. and Gorin, V. (2016). Fluctuations of particle systems determined by Schur generating functions. Available at arXiv:1604.01110.

[8] Bufetov, A. and KNIZEL, A. (2016). Asymptotics of random domino tilings of rectangular Aztec diamonds. Available at arXiv:1604.01491. 
[9] Chhita, S. and Johansson, K. (2016). Domino statistics of the two-periodic Aztec diamond. Adv. Math. 294 37-149.

[10] ChHita, S. and Young, B. (2014). Coupling functions for domino tilings of Aztec diamonds. Adv. Math. 259 173-251.

[11] Cohn, H., Kenyon, R. and Propp, J. (2001). A variational principle for domino tilings. J. Amer. Math. Soc. 14 297-346. MR1815214

[12] Corwin, I. and HAMmond, A. (2014). Brownian Gibbs property for Airy line ensembles. Invent. Math. 195 441-508.

[13] DE TILIÈRE, B. (2007). Scaling limit of isoradial dimer models and the case of triangular quadri-tilings. Ann. Inst. Henri Poincaré Probab. Stat. 43 729-750.

[14] Di Francesco, P. and Soto-Garrido, R. (2014). Arctic curves of the octahedron equation. J. Phys. A 47 1751-8113. MR3228361

[15] DubÉDAT, J. (2015). Dimers and families of Cauchy-Riemann operators I. J. Amer. Math. Soc. 28 1063-1167.

[16] DubÉdat, J. and Gheissari, R. (2015). Asymptotics of height change on toroidal Temperleyan dimer models. J. Stat. Phys. 159 75-100.

[17] Duits, M. (2013). Gaussian free field in an interlacing particle system with two jump rates. Comm. Pure Appl. Math. 66 600-643. MR3020314

[18] Duits, M. (2015). On global fluctuations for non-colliding processes. Available at arXiv: 1510.08248.

[19] Ferrari, P. L. and Spohn, H. (2003). Step fluctuations for a faceted crystal. J. Stat. Phys. 113 1-46. MR2012974

[20] Gorin, V. (2017). Bulk universality for random lozenge tilings near straight boundaries and for tensor products. Comm. Math. Phys. 354 317-344. MR3656520

[21] Johansson, K. (2003). Discrete polynuclear growth and determinantal processes. Comm. Math. Phys. 242 277-329.

[22] Johansson, K. (2005). The Arctic circle boundary and the Airy process. Ann. Probab. 33 1-30. MR2118857

[23] Johansson, K. (2017). Edge fluctuations of limit shapes. Available at arXiv:1704.06035.

[24] Kenyon, R. (1997). Local statistics of lattice dimers. Ann. Inst. Henri Poincaré Probab. Stat. 33 591-618. MR1473567

[25] Kenyon, R. (2000). Conformal invariance of domino tiling. Ann. Probab. 28 759-795. MR1782431

[26] Kenyon, R. (2001). Dominos and the Gaussian free field. Ann. Probab. 29 1128-1137. MR1872739

[27] Kenyon, R. (2009). Lectures on dimers. In Statistical Mechanics. IAS/Park City Math. Ser. 16 191-230. Amer. Math. Soc., Providence, RI. MR2523460

[28] Kenyon, R. and Okounkov, A. (2007). Limit shapes and the complex Burgers equation. Acta Math. 199 263-302.

[29] Kenyon, R., Okounkov, A. and Sheffield, S. (2006). Dimers and amoebae. Ann. of Math. (2) 163 1019-1056.

[30] Nienhuis, B., Hilhorst, H. J. and BlÖte, H. W. J. (1984). Triangular SOS models and cubic-crystal shapes. J. Phys. A 17 3559-3581.

[31] Okounkov, A. and Reshetikhin, N. (2003). Correlation function of Schur process with application to local geometry of a random 3-dimensional Young diagram. J. Amer. Math. Soc. 16 581-603. MR1969205

[32] Panova, G. (2015). Lozenge tilings with free boundaries. Lett. Math. Phys. 105 1551-1586. MR3406712

[33] Petrov, L. (2014). Asymptotics of random lozenge tilings via Gelfand-Tsetlin schemes. Probab. Theory Related Fields $160429-487$. 
[34] Petrov, L. (2015). Asymptotics of uniformly random lozenge tilings of polygons. Gaussian free field. Ann. Probab. 43 1-43. MR3298467

[35] Prähofer, M. and SPOHn, H. (2002). Scale invariance of the PNG droplet and the Airy process. J. Stat. Phys. 108 1071-1106.

[36] Thurston, W. P. (1990). Conway's tiling groups. Amer. Math. Monthly 97 757-773. MR1072815

V. BEFFARA

UNIV. GRENOBLE ALPES

CNRS

INSTITUT FOURIER

F-38000 GRENOBLE

FRANCE

E-MAIL: vincent.beffara@univ-grenoble-alpes.fr

\section{S. CHHITA}

Department of Mathematical SCIEnCES DURHAM UNIVERSITY

DURHAM

DH1 3LE

UK

E-MAIL: sunil.chhita@durham.ac.uk

\section{K. JOHANSSON}

DEPARTMENT OF MATHEMATICS

ROYAL INSTITUTE OF TECHNOLOGY KTH

LINSTEDTSVÄGEN 25

STOCKHOLM SE10044

SWEDEN

E-MAIL: kurtj@kth.se 